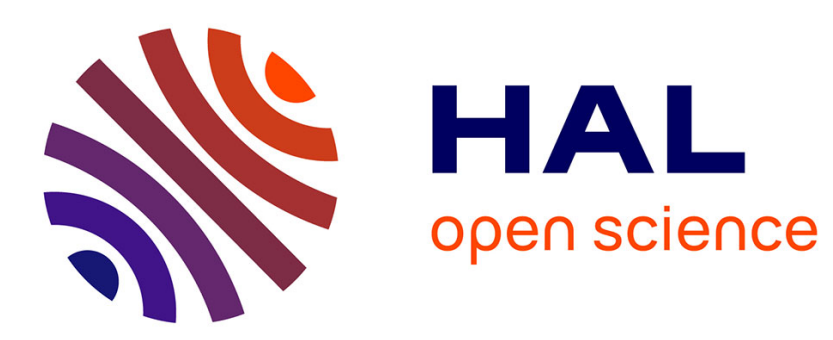

\title{
A verification theorem for optimal stopping problems with expectation constraints
}

\author{
Stefan Ankirchner, Maike Klein, Thomas Kruse
}

\section{To cite this version:}

Stefan Ankirchner, Maike Klein, Thomas Kruse. A verification theorem for optimal stopping problems with expectation constraints. 2015. hal-01229024

\section{HAL Id: hal-01229024 \\ https://hal.science/hal-01229024}

Preprint submitted on 16 Nov 2015

HAL is a multi-disciplinary open access archive for the deposit and dissemination of scientific research documents, whether they are published or not. The documents may come from teaching and research institutions in France or abroad, or from public or private research centers.
L'archive ouverte pluridisciplinaire HAL, est destinée au dépôt et à la diffusion de documents scientifiques de niveau recherche, publiés ou non, émanant des établissements d'enseignement et de recherche français ou étrangers, des laboratoires publics ou privés. 


\title{
A verification theorem for optimal stopping problems with expectation constraints
}

\author{
Stefan Ankirchner * Maike Klein $^{\dagger} \quad$ Thomas Kruse $^{\ddagger}$
}

November 16, 2015

We consider the problem of optimally stopping a continuous-time process with a stopping time satisfying a given expectation cost constraint. We show, by introducing a new state variable, that one can transform the problem into an unconstrained control problem and hence derive a dynamic programming principle. We characterize the value function in terms of the dynamic programming equation, which turns out to be an elliptic, fully non-linear partial differential equation of second order. We prove a classical verification theorem and illustrate its applicability with several examples.

\section{Introduction}

Let $\left(X_{t}\right)_{t \in \mathbb{R}_{+}}$be an $n$-dimensional stochastic state process that satisfies a stochastic differential equation driven by a $d$-dimensional Brownian motion $W$. Denote by $\left(\mathcal{F}_{t}\right)$ the filtration that is generated by $W$ and extended by null sets so as to satisfy the usual conditions. Let $f: \mathbb{R}^{n} \rightarrow \mathbb{R}$ be a payoff function and $H_{t}=\int_{0}^{t} h\left(X_{s}\right) d s$ an increasing cost process, where $h: \mathbb{R}^{n} \rightarrow(0, \infty)$. We denote by $\mathcal{S}(T)$ the set of $\left(\mathcal{F}_{t}\right)$-stopping times satisfying the constraint $E\left[H_{\tau}\right] \leq T \in \mathbb{R}_{+}$. In this article we consider the optimal stopping problem

$$
\text { maximize } E\left[f\left(X_{\tau}\right)\right] \quad \text { subject to } \tau \in \mathcal{S}(T) \text {. }
$$

\footnotetext{
*Stefan Ankirchner, Institute for Mathematics, University of Jena, Ernst-Abbe-Platz 2, 07745 Jena, Germany. Email: s.ankirchner@uni-jena.de, Phone: +49 (0)3641 946275.

${ }^{\dagger}$ Maike Klein, Institute for Mathematics, University of Jena, Ernst-Abbe-Platz 2, 07745 Jena, Germany. Email: maike.klein@uni-jena.de, Phone: +49 (0)3641 946186.

${ }^{\ddagger}$ Thomas Kruse, Faculty of Mathematics, University of Duisburg-Essen, Thea-Leymann-Str. 9, 45127 Essen, Germany. Email: thomas.kruse@uni-due.de, Phone: +49 (0)201 1833911.
} 
By choosing $h(t)=1$ for all $t \in \mathbb{R}_{+}$, we obtain as a special case the stopping problem over all stopping times with the expectation constraint $E\left[H_{\tau}\right]=$ $E[\tau] \leq T$

The problem (0.1) captures situations where there is an average time/cost limit for any stopping rule. Whenever a stopping rule is applied repeatedly, an average constraint seems to be more appropriate than a sharp constraint requiring that any stopping time $\tau$ satisfies $H_{\tau} \leq T$, a.s. Besides, an expectation constraint on the stopping time is a suitable counterpart for a gain functional that is itself an expectation.

What makes the stopping problem (0.1) difficult is that there is no simple dependence of the constraint on time. The expectation constraint has to be turned into a scenario-dependent constraint. A first attempt to eliminate the constraint is to follow a Lagrange approach and to consider, for every $\lambda>0$, the unconstrained stopping problem

$$
w(\lambda)=\sup \left\{E\left[f\left(X_{\tau}\right)-\lambda(\tau-T)\right]: \tau \text { stopping time with } E[\tau]<\infty\right\} .
$$

Notice that (0.2) is an infinite horizon stopping problem that does not involve a discount factor. Therefore it is often impossible to characterize $w$ as the unique solution of a dynamic programming equation (cf. Section $6)$. Disregard this for a moment and assume that we can identify an optimal stopping time $\tau^{*}(\lambda)$ for $(0.2)$ and that $w$ is absolutely continuous with $\frac{\partial w}{\partial \lambda}(\lambda)=-E\left[\tau^{*}(\lambda)\right]$. If there exists $\hat{\lambda}$ such that $\frac{\partial w}{\partial \lambda}(\widehat{\lambda})=-T$, then the stopping time $\tau^{*}(\widehat{\lambda})$ is optimal for the original problem (0.1). It can happen, however, that the function $w$ is not absolutely continuous (see Section 6 for an example). Even if $w$ is differentiable, then it can be involved and errorprone to invert the derivative $\frac{\partial w}{\partial \lambda}$ and to determine the appropriate Lagrange multiplier $\widehat{\lambda}$.

In this article we propose a new approach for solving stopping problems of the type (0.1). Our basic idea is to extend the state space by the conditional expectation process of $H_{\tau}$. Assuming a Brownian set-up, the predictable representation property allows to interpret the new state variable as a martingale with controlled diffusion coefficient. One can thus transform the stopping problem (0.1) into a problem with a controlled state and time horizon. The advantage of the transformed problem is that it allows to formulate a dynamic programming principle (DPP). With a DPP at hand, we can characterize the value function $V(T, x)=\sup \left\{E\left[f\left(X_{\tau}\right)\right]: \tau \in \mathcal{S}(T)\right.$ and $\left.X_{0}=x\right\}$ as a solution of the dynamic programming equation (DPE). In order to obtain a classical verification theorem, we consider also the auxiliary stopping problem $U(T, x)=\sup \left\{E\left[f\left(X_{\tau}\right)\right]: X_{0}=x, \tau\right.$ a stopping time with $\left.E\left[H_{\tau}\right]=T\right\}$. One can show that $V(T, x)=U(T \wedge \tilde{T}(x), x)$, where $\tilde{T}(x)=\inf \{T \geq 0$ : $\left.\frac{\partial U}{\partial T}(T, x) \leq 0\right\}$. Consequently, $U$ fully determines $V$. The DPE for $U$ turns 
out to be the partial differential equation (PDE)

$$
h(x) U_{T}(T, x)-\mathcal{L} U(T, x)+\frac{\left|\sigma^{\top}(x) \cdot \nabla_{x} U_{T}(T, x)\right|^{2}}{2 U_{T T}(T, x)}=0,
$$

with initial condition $U(0, x)=f(x)$; here $\mathcal{L}$ is the generator of $X$ and $\sigma$ its diffusion matrix. We give sufficient conditions for the value function $U$ to be a solution of (0.3). Moreover, we provide a verification theorem that allows to verify whether a solution of (0.3) coincides with $U$. Since $V$ is determined by $U$, this allows further to identify an optimal stopping time for the original problem (0.1).

If no closed-form solution is at hand, then one can try to solve the PDE (0.3) numerically. Related is the question whether there is a comparison principle for viscosity solutions. We do not discuss these issues in the present article, but leave them for future research.

The idea to extend the state space by a conditional expectation process in order to make a constraint more tangible can be found already in the control literature. Bouchard, Elie and Touzi [2] consider the problem of attaining a possibly stochastic target with a given probability. They extend the state space by a conditional probability process in order to reduce the problem to a standard stochastic target problem. Bokanowski, Picarelli and Zidani [1] reformulate a stochastic control problem with a state constraint as a target problem by introducing a conditional expectation process as a new controlled variable.

There are only few articles in the literature that deal with stopping problems of the type (0.1). Kennedy [7] considers the problem of stopping a discrete time process with the constraint that the expectation of any stopping time is bounded by some given constant. He uses Lagrangian techniques for determining optimal stopping rules. Horiguchi [5] considers optimal stopping of a finite state process that, in addition, can be controlled with finitely many actions. Optimal stopping rules satisfying an expectation constraint are determined with mathematical programming techniques. Palczewski and Stettner [8] consider an undiscounted optimal stopping problem with infinite time horizon of the type (0.3) under the additional assumption that $X$ is an ergodic, time-homogeneous weak Feller process. They state sufficient conditions guaranteeing that the set of stopping times can be restricted to those with bounded expectation. This boundary is in general not global but depends on the initial value of $X$.

The paper is organized as follows. Section 1 states the precise assumptions. In Section 2 we establish a one-to-one correspondence between the set $\mathcal{S}(T)$ and a class of controlled martingales. The correspondence allows us to transform the constrained stopping problem so that one can formulate a dynamic programming principle (see Section 3). Section 4 deals with the dynamic programming equation and Section 5 provides a classical verification 
theorem. In Section 6 we briefly compare the new method with the Lagrange approach. Finally, in Section 7 we discuss several examples illustrating the scope of our results.

\section{Optimal stopping with expectation constraints}

Let $\left(W_{t}\right)_{t \geq 0}$ be a $d$-dimensional Brownian motion on a probability space $(\Omega, \mathcal{F}, P)$ and denote by $\left(\mathcal{F}_{t}\right)_{t \geq 0}$ its augmented natural filtration. Let $\mu$ : $\mathbb{R}^{n} \rightarrow \mathbb{R}^{n}$ and $\sigma: \mathbb{R}^{n} \rightarrow \mathbb{R}^{n \times d}$ be Lipschitz-continuous functions and assume that for every $x \in \mathbb{R}^{n}$ the matrix $\left(\sigma \sigma^{\top}\right)(x) \in \mathbb{R}^{n \times n}$ is positive definite. Then there exists a unique $\mathbb{R}^{n}$-valued strong solution $\left(X_{t}^{x, r}\right)_{t \geq r}$ of the SDE

$$
d X_{t}^{x, r}=\mu\left(X_{t}^{x, r}\right) d t+\sigma\left(X_{t}^{x, r}\right) \cdot d W_{t}, \quad X_{r}^{x, r}=x,
$$

for every $r \in \mathbb{R}_{+}$and $x \in \mathbb{R}^{n}$. Moreover, recall that $X$ fulfills the strong Markov property, cf. [6], Chapter 5. by

Let $h: \mathbb{R}^{n} \rightarrow(0, \infty)$ be Borel-measurable and define the process $\left(H_{t}^{x, r}\right)_{t \geq 0}$

$$
H_{t}^{x, r}=\int_{r}^{r+t} h\left(X_{s}^{x, r}\right) d s
$$

for $x \in \mathbb{R}^{n}$ and $r \in \mathbb{R}_{+}$fixed. Denote by $\mathcal{T}(T)=\mathcal{T}(T, x)$ and $\mathcal{S}(T)=\mathcal{S}(T, x)$ the set of all $\left(\mathcal{F}_{t}\right)$-stopping times $\tau$ with $E\left[H_{\tau}^{x, 0}\right]=T$ resp. $E\left[H_{\tau}^{x, 0}\right] \leq T$. Note that Assumption (A) guarantees that $\mathcal{T}(T)$ is nonempty; e.g. the stopping time $\tau=\inf \left\{s \geq 0 \mid H_{s}^{x, 0}>T\right\}$ satisfies $H_{\tau}^{x, 0}=T$, and hence lies in $\mathcal{T}(T)$. In the following we sometimes refer to $\mathcal{T}(T)$ as the set of admissible stopping times.

Standing Assumption. Throughout we assume that for all $x \in \mathbb{R}^{n}$ and $r \in \mathbb{R}_{+}$

$$
H_{t}^{x, r}<\infty \text { and } \lim _{t \rightarrow \infty} H_{t}^{x, r}=\infty, P-\text { a.s. }
$$

Note that if $h$ is bounded and bounded away from zero, i.e. $h: \mathbb{R}^{n} \rightarrow[\delta, C]$ with $0<\delta<C$, then Condition (A) is satisfied.

In order to simplify notation, in the following we often write $X_{t}^{x}$ and $H_{t}^{x}$ instead of $X_{t}^{x, 0}$ resp. $H_{t}^{x, 0}$. In addition, if the starting value $x$ is clear from the context, we omit it.

For a measurable function $f: \mathbb{R}^{n} \rightarrow \mathbb{R}$ we consider the following optimal stopping problem with constraint function $h$

$$
V(T, x)=\sup _{\tau \in \mathcal{S}(T)} E\left[f\left(X_{\tau}^{x}\right)\right]
$$


where $T \in \mathbb{R}_{+}$and $x \in \mathbb{R}^{n}$. Here we use the convention that $E\left[f\left(X_{\tau}^{x}\right)\right]=-\infty$ if both the negative and the positive part of $f\left(X_{\tau}^{x}\right)$ have infinite expectation. Notice that by Assumption (A) every $\tau \in \mathcal{S}(T)$ is finite a.s. and hence $X_{\tau}^{x}$ is well-defined.

It turns out to be useful to study also the stopping problem with the equality constraint $E\left[H_{\tau}\right]=T$. We therefore introduce

$$
U(T, x)=\sup _{\tau \in \mathcal{T}(T)} E\left[f\left(X_{\tau}^{x}\right)\right]
$$

Observe that by the very definition the function $T \mapsto V(T, x)$ is non-decreasing. Let $\tilde{T}(x)$ be the infimum of all time points $T$ at which $T \mapsto V(T, x)$ is not increasing (set $\tilde{T}(x)=\infty$ if the mapping is strictly increasing everywhere). Then, for all $T<\tilde{T}(x)$ we have $V(T, x)=U(T, x)$. Suppose for a moment that $T \mapsto U(T, x)$ is concave. Then this implies that also $T \mapsto V(T, x)$ is concave. Consequently, if $\tilde{T}(x)<\infty$, then $V(T, x)=V(\tilde{T}(x), x)$ for all $T \geq \tilde{T}(x)$. This means that $V$ is completely determined by $U$, namely we have

$$
V(T, x)=U(T \wedge \tilde{T}(x), x), \quad(T, x) \in \mathbb{R}_{+} \times \mathbb{R}^{n} .
$$

In the next sections we transform problem (1.3) into a control problem with an extended state space and derive a DPE for $U$. Moreover, we provide a verification theorem that allows to check whether a solution of the DPE coincides with the value function $U$. The link (1.4) allows us then to identify the value function $V$ and to obtain an optimal stopping time for the original problem (1.2).

In the above derivation of (1.4) we have assumed that $T \mapsto U(T, x)$ is concave. Conveniently, concavity turns out to be a consequence of the verification theorem. Notice, however, that one can heuristically show concavity of $T \mapsto U(T, x)$ as follows: let $\tau_{1} \in \mathcal{T}\left(T_{1}\right)$ and $\tau_{2} \in \mathcal{T}\left(T_{2}\right)$. Flip a coin with probability $\alpha \in(0,1)$ for head, and choose $\tau_{1}$ if head and $\tau_{2}$ if tail appears. With the randomized stopping time we can show $V\left(\alpha T_{1}+(1-\alpha) T_{2}, x\right) \geq$ $\alpha V\left(T_{1}, x\right)+(1-\alpha) V\left(T_{2}, x\right)$.

\section{Every admissible stopping time is a first hitting time}

In this section we establish a one-to-one correspondence between the set of stopping times $\mathcal{T}(m)$ and a class of $\left(\mathcal{F}_{t}\right)$-martingales solving a specific type of SDE with initial value $m$, where $m \in \mathbb{R}_{+}$. This correspondence allows us to transform the stopping problems (1.2) and (1.3).

For every $\tau \in \mathcal{T}(m)$, the process $\left(M_{t}\right)_{0 \leq t \leq \infty}$ defined by

$$
M_{t}=E\left[H_{\tau} \mid \mathcal{F}_{t}\right]
$$


is a continuous $\left(\mathcal{F}_{t}\right)$-martingale with $M_{\infty}=H_{\tau}$ and $M_{0}=E\left[H_{\tau}\right]=m$. Thus, the martingale representation theorem implies

$$
M_{t}=m+\int_{0}^{t} \alpha_{s} \cdot d W_{s}
$$

where $\left(\alpha_{t}\right)_{t \geq 0}=\left(\alpha_{t}^{1}, \ldots, \alpha_{t}^{d}\right)_{t \geq 0} \in L_{l o c}^{2}(W)$, i.e. $\left(\alpha_{t}\right)_{t \geq 0}$ is measurable, $\left(\mathcal{F}_{t}\right)$ adapted and there exists a sequence $\left(\sigma_{n}\right)_{n \in \mathbb{N}}$ of $\left(\mathcal{F}_{t}\right)$-stopping times with $\sigma_{n} \nearrow \infty$ a.s. such that for all $n \in \mathbb{N}$

$$
E\left[\int_{0}^{\sigma_{n}}\left|\alpha_{s}\right|^{2} d s\right]<\infty .
$$

Then, the stopping time $\tau$ can be characterized as the first time when the process of conditional expectations falls below the process $H_{t}$.

Lemma 2.1. For an $\left(\mathcal{F}_{t}\right)$-stopping time $\tau$ such that $H_{\tau}$ is integrable and $M_{t}:=E\left[H_{\tau} \mid \mathcal{F}_{t}\right], t \geq 0$, we have

$$
\tau=\inf \left\{t \geq 0 \mid M_{t} \leq H_{t}\right\} .
$$

Proof. Notice that

$$
M_{t}=E\left[H_{\tau} \mid \mathcal{F}_{t}\right]=\mathbb{1}_{\{\tau \leq t\}} H_{\tau}+\mathbb{1}_{\{\tau>t\}} E\left[H_{\tau} \mid \mathcal{F}_{t}\right] .
$$

Therefore, we have $M_{t}=H_{\tau} \leq H_{t}$ on $\{\tau \leq t\}$, which implies $\tau \geq \inf \left\{t \geq 0 \mid M_{t} \leq H_{t}\right\}$.

On the other hand it holds true that

$$
\mathbb{1}_{\{\tau>t\}} H_{\tau} \geq \mathbb{1}_{\{\tau>t\}} H_{t}
$$

and, hence, on $\{\tau>t\}$ it follows from (2.1) that a.s.

$$
M_{t}=E\left[H_{\tau} \mid \mathcal{F}_{t}\right]>H_{t} .
$$

From this we deduce that $\tau \leq \inf \left\{t \geq 0 \mid M_{t} \leq H_{t}\right\}$.

Lemma 2.1 implies that for $\tau \in \mathcal{T}(m)$ the following holds true

$$
\begin{array}{ll}
M_{s}>H_{s} & \text { for } s<\tau=\inf \left\{t \geq 0 \mid M_{t} \leq H_{t}\right\}, \\
M_{s}=M_{\tau} & \text { for } s \geq \tau=\inf \left\{t \geq 0 \mid M_{t} \leq H_{t}\right\} .
\end{array}
$$

Therefore, the process $M$ satisfies

$$
d M_{t}=\mathbb{1}_{\{\tau>t\}} \alpha_{t} \cdot d W_{t}=\mathbb{1}_{\left\{\forall s \leq t: M_{s}>H_{s}\right\}} \alpha_{t} \cdot d W_{t} .
$$

On $\left\{M_{t}>H_{t}\right\}$ we have $t<\tau=\inf \left\{s \geq 0 \mid M_{s} \leq H_{s}\right\}$, which implies $M_{s}>H_{s}$ for all $s \leq t$. Therefore, it follows that

$$
d M_{t}=\mathbb{1}_{\left\{M_{t}>H_{t}\right\}} \alpha_{t} \cdot d W_{t}, \quad M_{0}=m .
$$

We have thus shown that any stopping time $\tau \in \mathcal{T}(m)$ coincides with the first time a process solving (2.2) hits $H_{t}$. Indeed, there is a one-to-one correspondence between $\left(\mathcal{F}_{t}\right)$-stopping times with $E\left[H_{\tau}\right]=m$ and processes $\left(M_{t}\right)$ satisfying $(2.2)$. To establish this correspondence we need the following lemma. 
Lemma 2.2. Let $\left(\alpha_{t}\right)_{t \geq 0}=\left(\alpha_{t}^{1}, \ldots, \alpha_{t}^{d}\right)_{t \geq 0} \in L_{l o c}^{2}(W)$ and $m \in \mathbb{R}_{+}$. Then there exists a unique solution $M$ of $(2.2)$. This solution is a non-negative martingale.

Proof. Let $\left(\alpha_{t}\right)_{t \geq 0} \in L_{l o c}^{2}(W), m \in \mathbb{R}_{+}$and $Y_{t}=m+\int_{0}^{t} \alpha_{s} \cdot d W_{s}$. Then,

$$
\tau=\inf \left\{t \geq 0 \mid Y_{t} \leq H_{t}\right\}
$$

defines an $\left(\mathcal{F}_{t}\right)$-stopping time and the stopped process $M_{t}:=Y_{t \wedge \tau}$ satisfies

$$
\begin{aligned}
d M_{t} & =\mathbb{1}_{\{\tau>t\}} \alpha_{t} \cdot d W_{t} \\
& =\mathbb{1}_{\left\{\forall s \leq t: Y_{s}>H_{s}\right\}} \alpha_{t} \cdot d W_{t} \\
& =\mathbb{1}_{\left\{\forall s \leq t: M_{s}=Y_{s} \text { and } M_{s}>H_{s}\right\}} \alpha_{t} \cdot d W_{t} .
\end{aligned}
$$

Notice that $M_{t}>H_{t}$ implies $M_{s}>H_{s}$ for all $s \leq t$ he definition of $\tau$. Hence,

$$
\left\{\forall s \leq t: M_{s}=Y_{s} \text { and } M_{s}>H_{s}\right\}=\left\{M_{t}>H_{t}\right\}
$$

and $\left(M_{t}\right)$ solves $(2.2)$.

We next show that $M$ is the unique solution of (2.2). To this end let $\left(N_{t}\right)$ be another solution of (2.2). Let $\rho=\inf \left\{t \geq 0 \mid N_{t} \leq H_{t}\right\}$. Then $N_{t \wedge \rho}=m+\int_{0}^{\rho \wedge t} \alpha_{s} \cdot d W_{s}$, which implies $\rho=\tau$ and hence $N_{t}=M_{t}$ on $[0, \tau]$. After $\tau$ we have $N_{t}=N_{\tau}=M_{\tau}=M_{t}$. Therefore, $N=M$.

We now show that $M$ is a martingale. First notice that by definition $M$ is a continuous, non-negative local martingale. Hence, it is a supermartingale and the limit

$$
M_{\infty}=\lim _{t \rightarrow \infty} M_{t}
$$

exists almost surely with $M_{\infty} \in L^{1}(\Omega)$.

Note that Fatou's lemma implies $E\left[M_{\infty}\right] \leq m$. In order to prove that $M$ is a true martingale, it suffices to show that $E\left[M_{\infty}\right] \geq m$. To this end observe that monotone convergence implies

$$
E\left[M_{\infty}\right]=\lim _{n \rightarrow \infty} E\left[M_{\infty} \mathbb{1}_{\left\{M_{\infty} \leq n\right\}}\right] .
$$

Define the stopping times $\tau_{n}$ by

$$
\tau_{n}=\inf \left\{t \geq 0 \mid M_{t} \leq H_{t} \text { or } M_{t} \geq n\right\}
$$

Then, $\tau_{n} \leq \tau$ almost surely. The stopped processes $\left(M_{t \wedge \tau_{n}}\right)_{t \geq 0}$ are martingales with

$$
E\left[M_{\tau_{n}}\right]=\lim _{t \rightarrow \infty} E\left[M_{\tau_{n} \wedge t}\right]=m
$$


by the dominated convergence theorem. Notice that $\tau_{n}=\tau$ on $\left\{M_{\tau} \leq n\right\}$. This, together with $M_{\infty}=M_{\tau}$, gives

$$
\begin{aligned}
E\left[M_{\infty} \mathbb{1}_{\left\{M_{\infty} \leq n\right\}}\right] & =E\left[M_{\tau_{n}} \mathbb{1}_{\left\{M_{\tau} \leq n\right\}}\right] \\
& =E\left[M_{\tau_{n}}\right]-E\left[M_{\tau_{n}} \mathbb{1}_{\left\{M_{\tau}>n\right\}}\right] \\
& =m-n \mathbb{P}\left[M_{\tau}>n\right] \\
& \geq m-E\left[M_{\tau} \mathbb{1}_{\left\{M_{\tau}>n\right\}}\right] \\
& =m-E\left[M_{\infty} \mathbb{1}_{\left\{M_{\infty}>n\right\}}\right] .
\end{aligned}
$$

To sum up, we have

$$
E\left[M_{\infty}\right] \geq m
$$

and hence $M$ is a true martingale.

Let $\mathcal{M}(m)$ be the set of all solutions $M$ of $(2.2)$ with $\left(\alpha_{t}\right)_{t \geq 0} \in L_{l o c}^{2}(W)$. The results obtained so far show that one can identify $\mathcal{T}(m)$ with $\mathcal{M}(m)$.

Proposition 2.3. There is a one-to-one correspondence between $\mathcal{T}(m)$ and $\mathcal{M}(m)$ given by

$$
M_{t}=E\left[H_{\tau} \mid \mathcal{F}_{t}\right], t \geq 0, \quad \text { and } \quad \tau=\inf \left\{t \geq 0 \mid M_{t} \leq H_{t}\right\},
$$

where $\tau \in \mathcal{T}(m)$ and $M \in \mathcal{M}(m)$. Moreover, $H_{\tau}=M_{\tau}=M_{\infty}$.

Proof. The statements follow from Lemma 2.1 and 2.2, and the discussion preceding Lemma 2.2.

Remark 2.4. To emphasize the dependence on $\alpha$ and $m$, in the following we often write $M^{\alpha, m}$ instead of $M$.

Example 2.5. Let $\left(W_{t}\right)_{t \geq 0}$ be a Brownian motion in $\mathbb{R}^{d}$. For $x \in \mathbb{R}^{d}$ let $X_{t}^{x}=x+W_{t}, t \geq 0$, and the constraint function $h$ is given by $h(y)=1, y \in$ $\mathbb{R}^{d}$, hence, we have $H_{t}=t$. Denote by $\tau_{R}$ the first exit time of the ball around 0 with radius $R>|x|$, i.e. $\tau_{R}=\inf \left\{t \geq 0|| X_{t}^{x} \mid \geq R\right\}$. Then, the expected value of $\tau_{R}$ is given by

$$
E\left[\tau_{R}\right]=\frac{R^{2}-|x|^{2}}{d}
$$

see Chapter 4.2.E in [6]. Hence, on $\left\{\tau_{R}>t\right\}$ the process of conditional expectations $M_{t}$ is given by

$$
\begin{aligned}
M_{t}=E\left[\tau_{R} \mid \mathcal{F}_{t}\right] & =t+\frac{R^{2}-\left|X_{t}^{x}\right|^{2}}{d} \\
& =\frac{R^{2}-|x|^{2}}{d}+\frac{1}{d} \int_{0}^{t}-2 X_{s}^{x} \cdot d W_{s} .
\end{aligned}
$$

Thus, $\alpha_{s}=-2 X_{s}^{x} / d, s \geq 0$, with localizing sequence $\tau_{n}=n$ and we conclude that $\tau_{R}=\inf \left\{t \geq 0 \mid M_{t}^{\alpha,\left(R^{2}-|x|^{2}\right) / d} \leq t\right\}$ from Proposition 2.3. 
In dimension 1 we can extend the above example to exit times of intervals $(a, b), a<x<b$, instead of intervals $(-R, R)$ with $R>|x|$.

Example 2.6. For $x \in \mathbb{R}$ let $X_{t}^{x}=x+W_{t}, t \geq 0$, where $\left(W_{t}\right)_{t \geq 0}$ is a one-dimensional Brownian motion. Again let $h(y)=1, y \in \mathbb{R}$, and, thus, $H_{t}=t$. The first exit time $\tau$ of an interval $(a, b), a<x<b$, has expectation $(b-x)(x-a)$. The associated process of conditional expectations $M_{t}$ on $\left\{M_{t}>t\right\}$ is given by

$$
M_{t}=(b-x)(x-a)+\int_{0}^{t} \alpha_{s} d W_{s}
$$

with $\alpha_{s}=-2 X_{s}^{x}+b+a, s \geq 0$, and $\left(\alpha_{s}\right) \in L_{l o c}^{2}(W)$.

\section{The stopping problem as an optimal control problem}

The one-to-one correspondence of Proposition 2.3 allows to reformulate the optimal stopping problems (1.2) and (1.3) as optimal control problems. To do so, observe first that $t \mapsto H_{t}^{x, r}$ is continuous and strictly increasing almost surely. Moreover, note that Condition (A) guarantees that the pathwise inverse of $H^{x, r}$,

$$
G^{x, r}(t)=\inf \left\{s \geq 0 \mid H_{s}^{x, r} \geq t\right\},
$$

is defined for $t, r \in \mathbb{R}_{+}$and $x \in \mathbb{R}^{n}$. In order to simplify notation, we write $G^{x}(t)$ instead of $G^{x, 0}(t)$.

Notice that if $\tau \in \mathcal{T}(m)$ and $M \in \mathcal{M}(m)$ such that $H_{\tau}=M_{\infty}$, then $\tau=G\left(M_{\infty}\right)$. Therefore, by Proposition 2.3 we have

$$
V(T, x)=\sup \left\{E\left[f\left(X_{G^{x}\left(M_{\infty}^{\alpha, m}\right)}^{x}\right)\right] \mid m \in[0, T], \alpha \in L_{l o c}^{2}(W)\right\}
$$

and

$$
U(T, x)=\sup \left\{E\left[f\left(X_{G^{x}\left(M_{\infty}^{\alpha, T}\right)}^{x}\right)\right] \mid \alpha \in L_{l o c}^{2}(W)\right\}
$$

where

$$
d M_{t}^{\alpha, m}=\mathbb{1}_{\left\{M_{t}^{\alpha, m}>H_{t}\right\}} \alpha_{t} \cdot d W_{t}, \quad M_{0}^{\alpha, m}=m .
$$

At a first glance, the reformulations $(\mathcal{V})$ and $(\mathcal{U})$ look more cumbersome than the original formulations of the stopping problem. We show in the next section, however, that the new formulations allow to obtain a dynamic programming principle. 


\section{Derivation of a dynamic programming principle}

The aim of this section is to derive a dynamic programming principle (DPP) for $U$ under suitable measurablility assumptions. For the derivation we need that the value function $U$ is finite. We start, therefore, with a subsection providing sufficient conditions for finiteness.

\subsection{Finiteness of the value functions}

Note that if the payoff function $f$ is bounded, then the value functions are also bounded. We next give a more general condition, in the one-dimensional case $n=d=1$, guaranteeing that the value functions $V$ and $U$ are finite.

Let $d=n=1$ and denote by $\mathcal{I} \subseteq \mathbb{R}$ the interior of the state space of $X$. By assumption we have $\sigma^{2}(x)>0$ for all $x \in \mathcal{I}$. Furthermore, we assume that $(1+|\mu(x)|) / \sigma^{2}(x)$ is locally integrable on $\mathcal{I}$ (see conditions (ND)' and $(\mathrm{LI})^{\prime}$ in Section 5.5.C of [6]). Let $X^{x}$ be a solution of (1.1) with $X_{0}^{x}=x$ and define the scale function $s$ by

$$
s_{x}(y)=\int_{x}^{y} \exp \left(-\int_{x}^{z} \frac{2 \mu(w)}{\sigma^{2}(w)} d w\right) d z .
$$

Then the process $Z_{t}:=s_{x}\left(X_{t}^{x}\right), t \geq 0$, is a local martingale starting in 0 with

$$
d Z_{t}=\eta\left(Z_{t}\right) d W_{t}
$$

where $\eta=\left(s_{x}^{\prime} \sigma\right) \circ s_{x}^{-1}$. Hence we can convert the optimal stopping problem with reward function $f$ for the process $X$ into an optimal stopping problem with reward function $f \circ s^{-1}$ for $Z$. Let

$$
q_{x}(y)=\int_{0}^{y} \int_{0}^{z} \frac{2 h\left(s_{x}^{-1}(w)\right)}{\eta^{2}(w)} d w d z .
$$

In the following we show that if $f \circ s_{x}^{-1}$ is bounded from above by $q_{x}, x \in \mathcal{I}$, then $V(T, x)<\infty$ for all $T \in \mathbb{R}_{+}$. More precisely,

Proposition 3.1. Let $x \in \mathcal{I}$. If there exists $C>0$ such that $f\left(s_{x}^{-1}(y)\right) \leq$ $C\left(1+q_{x}(y)\right)$ for all $y \in \overline{\mathcal{I}}$, then $U(T, x) \leq V(T, x)<\infty$. If, in addition $\left|E\left[f\left(X_{G(T)}^{x}\right)\right]\right|<\infty$ for all $x \in \mathbb{R}^{n}$, then $|U(T, x)|<\infty$.

Proof. First notice that the definition of $V$ implies $V(T, x) \geq f(x)$ for all $x \in \mathcal{I}$.

The function $q_{x}$ is convex, because $q_{x}^{\prime \prime}(y)=2 h\left(s_{x}^{-1}(y)\right) / \eta^{2}(y)>0$ and hence we can apply Itô's formula for convex functions to show that $q_{x}\left(Z_{t}\right)-H_{t}^{x}$, $t \geq 0$, is a local martingale. Moreover, we have $E\left[q_{x}\left(Z_{\tau}\right)\right] \leq T$ for all 
$\tau \in \mathcal{S}(T)$ : Let $\left(\tau_{n}\right)_{n \in \mathbb{N}}$ be a localizing sequence for $Z$. Then Fatou's lemma and the monotone convergence theorem imply that

$E\left[q_{x}\left(Z_{\tau}^{x}\right)\right]=E\left[\liminf _{n \rightarrow \infty} q_{x}\left(Z_{\tau \wedge \tau_{n}}^{x}\right)\right] \leq \liminf _{n \rightarrow \infty} E\left[q_{x}\left(Z_{\tau \wedge \tau_{n}}^{x}\right)\right]=\liminf _{n \rightarrow \infty} E\left[H_{\tau \wedge \tau_{n}}^{x}\right]=E\left[H_{\tau}^{x}\right] \leq T$

Therefore, we have for $T \in \mathbb{R}_{+}$

$$
\begin{aligned}
U(T, x) \leq V(T, x) & =\sup _{\tau \in \mathcal{S}(T)} E\left[f\left(X_{\tau}^{x}\right)\right] \\
& =\sup _{\tau \in \mathcal{S}(T)} E\left[f\left(s_{x}^{-1}\left(Z_{\tau}\right)\right)\right] \\
& \leq\left(1+\sup _{\tau \in \mathcal{S}(T)} E\left[q_{x}\left(Z_{\tau}\right)\right]\right) \\
& \leq C(1+T) .
\end{aligned}
$$

If, in addition, $E\left[f\left(X_{G(T)}^{x}\right)\right]<\infty$, then $|U(T, x)|<\infty$.

The following example shows that the condition from Proposition 3.1 is sharp if $X$ is a Brownian motion.

Example 3.2. For a one-dimensional Brownian motion $W$ we have $q_{0}(y)=$ $y^{2}$. Consider the optimal stopping problem (1.2) for $f(y)=|y|^{2+\varepsilon}, \varepsilon>0$, constraint function $h(x)=1$, i.e. $H_{t}=t$, and the Brownian motion $W$. For every $T>0$ the first time $H(a,-T / a), a>0$, when $W$ hits $a$ or $-T / a$ has expectation $T$. Hence,

$V(T, 0) \geq U(T, 0) \geq \sup _{a>0} E\left[f\left(W_{H(a,-T / a)}\right)\right]=\sup _{a>0}\left\{a^{2+\varepsilon} \frac{T}{a^{2}+T}+\frac{a^{2}}{a^{2+\varepsilon}} \frac{T^{2+\varepsilon}}{a^{2}+T}\right\}=\infty$.

\subsection{Dynamic programming principle for $U$}

In this subsection we prove a DPP for $U$ under the following assumptions.

\section{Assumption.}

(U0) $|U(T, x)|<\infty$ for all $x \in \mathbb{R}^{n}, T \in \mathbb{R}_{+}$.

(U1) $U$ is measurable.

(U2) For all $T \geq 0$, any family $\left(\theta^{\alpha}\right)_{\alpha \in L_{l o c}^{2}(W)}$ of $\left(\mathcal{F}_{t}\right)$-stopping times, for any $\varepsilon>0$ and $\omega \in \Omega$ with $M_{\theta^{\alpha}}^{\alpha, T}(\omega)>H_{\theta^{\alpha}}^{x}(\omega)$ there exists $\beta=\beta^{\varepsilon, \omega} \in$ $L_{l o c}^{2}(W)$ with

$$
E\left[f\left(X_{G_{\theta^{\alpha}, \theta^{\alpha}}^{x}\left(\bar{M}_{\infty}\right)}^{X_{x}^{x}, \theta^{\alpha}}\right) \mid \mathcal{F}_{\theta^{\alpha}}\right] \geq U\left(M_{\theta^{\alpha}}^{\alpha, T}(\omega)-H_{\theta^{\alpha}}^{x}(\omega), X_{\theta^{\alpha}}^{x}(\omega)\right)-\varepsilon
$$


where $\bar{M}_{\infty}=\bar{M}_{\infty}^{\varepsilon, \omega}=M_{\infty}^{\beta, M_{\theta^{\alpha}}^{\alpha, T}(\omega)-H_{\theta^{\alpha}}^{x}(\omega)}$, such that the process $\hat{\alpha}$ defined by

$$
\hat{\alpha}_{s}(\omega)= \begin{cases}\alpha_{s}(\omega) & \text { if } s \in\left[0, \theta^{\alpha}(\omega)\right] \text { or }\left(s>\theta^{\alpha}(\omega) \text { and } M_{\theta^{\alpha}}^{\alpha, T}(\omega) \leq H_{\theta^{\alpha}}^{x}(\omega)\right), \\ \beta_{s-\theta^{\alpha}}(\omega) & \text { if } s>\theta^{\alpha}(\omega) \text { and } M_{\theta^{\alpha}}^{\alpha, T}(\omega)>H_{\theta^{\alpha}}^{x}(\omega),\end{cases}
$$

satisfies $\hat{\alpha} \in L_{l o c}^{2}(W)$.

Proposition 3.3 (DPP for $U$ ). Let the Assumptions (U0) - (U2) be satisfied. For any family of $\left(\mathcal{F}_{t}\right)$-stopping times $\left(\theta^{\alpha}\right)_{\alpha \in L_{\text {loc }}^{2}(W)}$ we have

$$
U(T, x)=\sup _{\alpha \in L_{l o c}^{2}(W)} E\left[\mathbb{1}_{\left\{M_{\theta^{\alpha}}^{\alpha, T} \leq H_{\theta^{\alpha}}^{x}\right\}} f\left(X_{G^{x}\left(M_{\theta^{\alpha}}^{\alpha, T}\right)}^{x}\right)+\mathbb{1}_{\left\{M_{\theta^{\alpha}}^{\alpha, T}>H_{\theta^{\alpha}}^{x}\right\}} U\left(M_{\theta^{\alpha}}^{\alpha, T}-H_{\theta^{\alpha}}^{x}, X_{\theta^{\alpha}}^{x}\right)\right] .
$$

Remark 3.4. Using the upper- and lower-semicontinuous envelopes of $U$ one can formulate a weak DPP, in the fashion of [3], that does not require Assumption (U1) and the measurable selection assumption (U2).

Proof. Fix $(T, x) \in(0, \infty) \times \mathbb{R}^{n}$ and let $\left(\theta^{\alpha}\right)_{\alpha \in L_{l o c}^{2}(W)}$ be a family of $\left(\mathcal{F}_{t}\right)$ stopping times. Then for every $\alpha \in L_{l o c}^{2}(W)$ we have

$$
\begin{aligned}
E\left[f \left(X_{\left.\left.G^{x}\left(M_{\infty}^{\alpha, T}\right)\right)\right]}^{x}\right.\right. & =E\left[\mathbb{1}_{\left\{M_{\theta^{\alpha}}^{\alpha, T} \leq H_{\theta^{\alpha}}^{x}\right\}} f\left(X_{G^{x}\left(M_{\infty}^{\alpha, T}\right)}^{x}\right)+\mathbb{1}_{\left\{M_{\theta^{\alpha}}^{\alpha, T}>H_{\left.\theta^{\alpha}\right\}}^{x}\right.} f\left(X_{G^{x}\left(M_{\infty}^{\alpha, T}\right)}^{x}\right)\right] \\
& =E\left[\mathbb{1}_{\left\{M_{\theta^{\alpha}}^{\alpha, T} \leq H_{\theta^{\alpha}}^{x}\right\}} f\left(X_{G^{x}\left(M_{\theta^{\alpha}}^{\alpha, T}\right)}^{x}\right)+\mathbb{1}_{\left\{M_{\theta^{\alpha}}^{\alpha, T}>H_{\left.\theta^{\alpha}\right\}}^{x}\right.} f\left(X_{G^{x}\left(M_{\infty}^{\alpha, T}\right)}^{x}\right)\right],
\end{aligned}
$$

where we use that $M_{\infty}^{\alpha, T}$ and $M_{\theta^{\alpha}}^{\alpha, T}$ coincide on $\left\{M_{\theta^{\alpha}}^{\alpha, T} \leq H_{\theta^{\alpha}}^{x}\right\}$. By conditioning the second summand on $\mathcal{F}_{\theta^{\alpha}}$, the strong Markov property of $X^{x}$ implies

$$
\begin{aligned}
& E\left[\mathbb{1}_{\left\{M_{\theta^{\alpha}}^{\alpha, T}>H_{\theta^{\alpha}}^{x}\right\}} f\left(X_{G^{x}\left(M_{\infty}^{\alpha, T}\right)}^{x}\right)\right]=E\left[E\left[\mathbb{1}_{\left\{M_{\theta^{\alpha}}^{\alpha, T}>H_{\theta^{\alpha}}^{x}\right\}} f\left(X_{G^{x}\left(M_{\infty}^{\alpha, T}\right)}^{x}\right) \mid \mathcal{F}_{\theta^{\alpha}}\right]\right] \\
& =E\left[\mathbb{1}_{\left\{M_{\theta^{\alpha}}^{\alpha, T}>H_{\left.\theta^{\alpha}\right\}}^{x}\right.} E\left[f\left(X_{G^{x}\left(M_{\infty}^{\alpha, T}\right)-\theta^{\alpha}}^{X^{x}, \theta^{\alpha}}\right) \mid \mathcal{F}_{\theta^{\alpha}}\right]\right] \text {. }
\end{aligned}
$$

The expectation constraint conditioned on $\mathcal{F}_{\theta^{\alpha}}$ results in

$$
\begin{aligned}
E\left[H_{G^{x}\left(M_{\infty}^{X, T}\right)-\theta^{\alpha}}^{X_{\theta^{\alpha}}, \theta^{\alpha}} \mid \mathcal{F}_{\theta^{\alpha}}\right] & =E\left[\int_{\theta^{\alpha}}^{G^{x}\left(M_{\infty}^{\alpha, T}\right)} h\left(X_{s}^{X_{\theta^{\alpha}}^{x}, \theta^{\alpha}}\right) d s \mid \mathcal{F}_{\theta^{\alpha}}\right] \\
& =E\left[\int_{\theta^{\alpha}}^{G^{x}\left(M_{\infty}^{\alpha, T}\right)} h\left(X_{s}^{x}\right) d s \mid \mathcal{F}_{\theta^{\alpha}}\right] \\
& =E\left[H_{G^{x}\left(M_{\infty}^{\alpha, T}\right)}^{x}-H_{\theta^{\alpha}}^{x} \mid \mathcal{F}_{\theta^{\alpha}}\right] \\
& =E\left[M_{\infty}^{\alpha, T} \mid \mathcal{F}_{\theta^{\alpha}}\right]-H_{\theta^{\alpha}}^{x} \\
& =M_{\theta^{\alpha}}^{\alpha, T}-H_{\theta^{\alpha}}^{x}
\end{aligned}
$$


because $\left(M_{t}^{\alpha, T}\right)$ is a martingale (Lemma 2.2). Thus, the conditional expectation of the remaining constraint equals $M_{\theta^{\alpha}}^{\alpha, T}-H_{\theta^{\alpha}}^{x}$. Hence,

$U(T, x) \leq \sup _{\alpha \in L_{l o c}^{2}(W)} E\left[\mathbb{1}_{\left\{M_{\theta^{\alpha}}^{\alpha, T} \leq H_{\theta^{\alpha}}^{x}\right\}} f\left(X_{G^{x}\left(M_{\theta^{\alpha}}^{\alpha, T}\right)}^{x}\right)+\mathbb{1}_{\left\{M_{\theta^{\alpha}}^{\alpha, T}>H_{\theta^{\alpha}}^{x}\right\}} U\left(M_{\theta^{\alpha}}^{\alpha, T}-H_{\theta^{\alpha}}^{x}, X_{\theta^{\alpha}}^{x}\right)\right]$

where we use that $U$ is measurable (Assumption (U1)).

For the reverse inequality let $\alpha \in L_{l o c}^{2}(W)$. For every $\varepsilon>0$ and every $\omega \in \Omega$ with $M_{\theta^{\alpha}}^{\alpha, T}(\omega)>H_{\theta^{\alpha}}^{x}(\omega)$, choose $\beta=\beta^{\varepsilon, \omega} \in L_{\text {loc }}^{2}(W)$ such that (3.1) holds and such that the control $\hat{\alpha}$ defined in (3.2) is in $L_{l o c}^{2}(W)$, which exists by Assumption (U2). Thus,

$$
\begin{aligned}
& U(T, x) \geq E\left[f\left(X_{G^{x}\left(M_{\infty}^{\hat{\alpha}, T}\right)}^{x}\right)\right] \\
& =E\left[\mathbb{1}_{\left\{M_{\theta^{\alpha}}^{\hat{\alpha}, T} \leq H_{\theta^{\alpha}}^{x}\right\}} f\left(X_{G^{x}\left(M_{\theta^{\alpha}}^{\hat{\alpha}, T}\right)}^{x}\right)+\mathbb{1}_{\left\{M_{\theta^{\alpha}}^{\hat{\alpha}, T}>H_{\theta^{\alpha}}^{x}\right\}} E\left[f\left(X_{G^{x}\left(M_{\infty}^{\hat{\alpha}, T}\right)}^{x}\right) \mid \mathcal{F}_{\theta^{\alpha}}\right]\right] \\
& =E\left[\mathbb{1}_{\left\{M_{\theta^{\alpha}}^{\alpha, T} \leq H_{\left.\theta^{\alpha}\right\}}^{x}\right.} f\left(X_{G^{x}\left(M_{\theta^{\alpha}}^{\alpha, T}\right)}^{x}\right)+\mathbb{1}_{\left\{M_{\theta^{\alpha}}^{\alpha, T}>H_{\theta^{\alpha}}^{x}\right\}} E\left[f\left(X_{G^{x}\left(M_{\infty}^{\alpha, T}\right)-\theta^{\alpha}}^{X_{\theta^{\alpha}}^{x}, \theta^{\alpha}}\right) \mid \mathcal{F}_{\theta^{\alpha}}\right]\right]
\end{aligned}
$$

by the strong Markov property and the fact that $M_{s}^{\hat{\alpha}, T}(\omega)=M_{s}^{\alpha, T}(\omega)$ for all $s \in\left[0, \theta^{\alpha}(\omega)\right]$. Notice that $G^{x}\left(M_{\infty}^{\hat{\alpha}, T}\right)-\theta^{\alpha}$ and $G^{X_{\theta}^{\alpha}, \theta^{\alpha}}\left(\bar{M}_{\infty}\right)=G^{X_{\theta^{\alpha}}^{x}, \theta^{\alpha}}\left(M_{\infty}^{\beta, M_{\theta^{\alpha}}^{\alpha, T}-H_{\theta^{\alpha}}^{x}}\right)$ both conditioned on $\left\{M_{\theta^{\alpha}}^{\alpha, T}>H_{\theta^{\alpha}}^{x}\right\}$ have the same distribution. Therefore,

$$
\begin{aligned}
U(T, x) & \geq E\left[\mathbb{1}_{\left\{M_{\theta^{\alpha}}^{\alpha, T} \leq H_{\left.\theta^{\alpha}\right\}}^{x}\right.} f\left(X_{G^{x}\left(M_{\theta^{\alpha}}^{\alpha, T}\right)}^{x}\right)+\mathbb{1}_{\left\{M_{\theta^{\alpha}}^{\alpha, T}>H_{\theta^{\alpha}}^{x}\right\}} E\left[f\left(X_{G^{X_{\theta^{\alpha}}, \theta^{\alpha}}\left(\bar{M}_{\infty}\right)}^{X^{x}, \theta^{\alpha}}\right) \mid \mathcal{F}_{\theta^{\alpha}}\right]\right] \\
& \geq E\left[\mathbb{1}_{\left\{M_{\theta^{\alpha}}^{\alpha, T} \leq H_{\theta^{\alpha}}^{x}\right\}} f\left(X_{G^{x}\left(M_{\theta^{\alpha}}^{\alpha, T}\right)}^{x}\right)+\mathbb{1}_{\left\{M_{\theta^{\alpha}}^{\alpha, T}>H_{\theta^{\alpha}}^{x}\right\}}\left(U\left(M_{\theta^{\alpha}}^{\alpha, T}-H_{\theta^{\alpha}}^{x}, X_{\theta^{\alpha}}^{x}\right)-\varepsilon\right)\right],
\end{aligned}
$$

which implies a DPP for $U$ by the arbitrariness of $\alpha \in L_{l o c}^{2}(W)$ and $\varepsilon>0$.

\section{The dynamic programming equation for $U$}

The DPP from Proposition 3.3 allows to derive a dynamic programming equation for $U$. In order to do so, we denote by $\mathcal{L}$ the generator of the Markov process $X$, i.e.

$$
\mathcal{L} u(x)=\frac{1}{2} \sum_{i=1}^{n} \sum_{j=1}^{n} \frac{\partial^{2} u}{\partial x_{i} \partial x_{j}}(x) \sum_{l=1}^{d} \sigma_{i l}(x) \sigma_{j l}(x)+\sum_{i=1}^{n} b_{i}(x) \frac{\partial u}{\partial x_{i}}(x)
$$


for suitable functions $u \in \mathcal{C}^{2}\left(\mathbb{R}^{n}, \mathbb{R}\right)$. For a function $u \in \mathcal{C}^{2}\left((0, \infty) \times \mathbb{R}^{n}, \mathbb{R}\right)$ we use the following notation:

$$
\begin{aligned}
u_{T}(T, x) & =\frac{\partial u}{\partial T}(T, x), \\
u_{T T}(T, x) & =\frac{\partial^{2} u}{\partial T^{2}}(T, x), \\
u_{T x_{i}}(T, x) & =\frac{\partial^{2} u}{\partial T \partial x_{i}}(T, x), \\
\nabla_{x} u(T, x) & =\left(\frac{\partial u}{\partial x_{i}}(T, x)\right)_{i=1}^{n}, \\
\nabla_{x} u_{T}(T, x) & =\left(u_{T x_{i}}(T, x)\right)_{i=1}^{n}
\end{aligned}
$$

and for a matrix $A \in \mathbb{R}^{k \times l}, k, l \in \mathbb{N}$, its transpose is denoted by $A^{\top}$.

Proposition 4.1. Assume that $h$ is continuous. If $U \in \mathcal{C}^{2}\left((0, \infty) \times \mathbb{R}^{n}\right)$ and $U$ satisfies the DPP (3.3), then

1. $U$ is a supersolution to

$$
h(x) U_{T}(T, x)-\mathcal{L} U(T, x)+\frac{\left|\sigma^{\top}(x) \cdot \nabla_{x} U_{T}(T, x)\right|^{2}}{2 U_{T T}(T, x)}=0
$$

on $(0, \infty) \times \mathbb{R}^{n}$ with initial condition $U(0, x)=f(x)$. Moreover, $U$ is concave in $T$ and

$$
\left\{(T, x) \mid U_{T T}(T, x)=0\right\} \subseteq\left\{(T, x) \mid \nabla_{x} U_{T}(T, x)=0 \in \mathbb{R}^{n}\right\} .
$$

Here we set $\left|\sigma^{\top}(x) \cdot \nabla_{x} U_{T}(T, x)\right|^{2} / U_{T T}(T, x)=0$ if both the numerator and the denominator equal 0 .

2. If, in addition, $\left|\sigma^{\top} \cdot \nabla_{x} U_{T}\right|^{2} / U_{T T}$ is continuous on $(0, \infty) \times \mathbb{R}^{n}$, then $U$ is a solution to (4.1) on $\bar{A} \cup(0, \infty) \times\left\{x \in \mathbb{R}^{n} \mid \exists T>0:(T, x) \in\right.$ $\bar{A}\}$, where $\bar{A}$ denotes the closure of $A:=\left\{(T, x) \mid U_{T T}(T, x)<0\right\}$ in $(0, \infty) \times \mathbb{R}^{n}$

Proof. 1. The initial condition is satisfied, because $T=0$ is equivalent to stopping directly. In order to prove that $U$ is a supersolution of (4.1) let $(T, x) \in(0, \infty) \times \mathbb{R}^{n}$, write $X$ instead of $X^{x}$ in the following and consider the constant control $\alpha \equiv a$, with $a \in \mathbb{R}^{d}$. Then, $\alpha \in L_{l o c}^{2}(W)$. Let

$$
\theta^{a}:=\inf \left\{s \geq 0|| X_{s}-x \mid \geq 1 \text { or } M_{s}^{a, T}-H_{s} \notin[T / 2,2 T]\right\} .
$$


The DPP for $U$ and Itô's formula imply that for $t>0$

$$
\begin{aligned}
0 \geq & E\left[U\left(M_{t \wedge \theta^{a}}^{a, T}-H_{t \wedge \theta^{a}}, X_{t \wedge \theta^{a}}\right)-U(T, x)\right] \\
\geq E & {\left[\int_{0}^{t \wedge \theta^{a}}\left(-h\left(X_{s}^{x}\right) U_{T}+\mathcal{L} U+\frac{|a|^{2}}{2} U_{T T}+\left(\nabla_{x} U_{T}\right)^{\top} \cdot \sigma\left(X_{s}\right) \cdot a\right)\left(M_{s}^{a, T}-H_{s}, X_{s}\right) d s\right] } \\
& +E\left[\int_{0}^{t \wedge \theta^{a}}\left(U_{T}\left(M_{s}^{a, T}-H_{s}, X_{s}\right) a+\left(\nabla_{x} U\left(M_{s}^{a, T}-H_{s}, X_{s}\right)\right)^{\top} \cdot \sigma\left(X_{s}\right)\right) \cdot d W_{s}\right] .
\end{aligned}
$$

The stochastic integral has expectation 0 , because the integrand is bounded on the stochastic interval $\left[0, t \wedge \theta^{a}\right]$.

By the pathwise continuity of $X_{s}$ and $M_{s}^{a, T}$ and the boundedness of the integrand in the Lebesgue integral we obtain, after first dividing by $t$ and then taking the limit $t \downarrow 0$, that

$$
-h(x) U_{T}(T, x)+\mathcal{L} U(T, x)+\frac{|a|^{2}}{2} U_{T T}(T, x)+\left(\nabla_{x} U_{T}(T, x)\right)^{\top} \cdot \sigma(x) \cdot a \leq 0
$$

for all $a \in \mathbb{R}^{d}$. Thus,

$$
-h(x) U_{T}(T, x)+\mathcal{L} U(T, x)+\sup _{a \in \mathbb{R}^{d}}\left\{\frac{|a|^{2}}{2} U_{T T}(T, x)+\left(\nabla_{x} U_{T}(T, x)\right)^{\top} \cdot \sigma(x) \cdot a\right\} \leq 0 .
$$

In particular, the supremum is finite which shows the concavity of $U$ in $T$ and

$$
\begin{aligned}
\left\{(T, x) \mid U_{T T}(T, x)=0\right\} & \subseteq\left\{(T, x) \mid\left(\nabla_{x} U_{T}(T, x)\right)^{\top} \cdot \sigma(x)=0 \in \mathbb{R}^{d}\right\} \\
& =\left\{(T, x) \mid \nabla_{x} U_{T}(T, x)=0 \in \mathbb{R}^{n}\right\} .
\end{aligned}
$$

The last conclusion follows from the fact that $\left(\sigma \sigma^{\top}\right)(x)$ is positive definite. Inequality (4.3) simplifies to

$$
-h(x) U_{T}(T, x)+\mathcal{L} U(T, x)-\frac{\left|\sigma^{\top}(x) \cdot \nabla_{x} U_{T}(T, x)\right|^{2}}{2 U_{T T}(T, x)} \leq 0
$$

if $U_{T T}(T, x)<0$ resp. $-h(x) U_{T}(T, x)+\mathcal{L} U(T, x)$ if $U_{T T}(T, x)=0$. To conclude, $U$ is a supersolution to (4.1), if we set $\left|\sigma^{\top}(x) \cdot \nabla_{x} U_{T}(T, x)\right|^{2} / U_{T T}(T, x)=$ 0 in the case that both expressions are 0 .

2. In order to prove that $U$ is a subsolution to (4.1) on $\bar{A}$ if $\left|\sigma^{\top} \cdot \nabla_{x} U_{T}\right|^{2} / U_{T T}$ is continuous, we first assume that there exists $\left(T_{0}, x_{0}\right) \in(0, \infty) \times \mathbb{R}^{n}$ with $U_{T T}\left(T_{0}, x_{0}\right)<0$ and

$$
h\left(x_{0}\right) U_{T}\left(T_{0}, x_{0}\right)-\mathcal{L} U\left(T_{0}, x_{0}\right)+\frac{\left|\sigma^{\top}\left(x_{0}\right) \cdot \nabla_{x} U_{T}\left(T_{0}, x_{0}\right)\right|^{2}}{2 U_{T T}\left(T_{0}, x_{0}\right)}>0 .
$$


Define for $(T, x) \in(0, \infty) \times \mathbb{R}^{n}$

$$
\varphi(T, x)=U(T, x)+\left|T-T_{0}\right|^{4}+\left|x-x_{0}\right|^{4} .
$$

Then $\varphi \in \mathcal{C}^{2}\left((0, \infty) \times \mathbb{R}^{n}\right)$ and (4.4) holds also if $U$ is replaced with $\varphi$. Moreover, the continuity of the derivatives implies that

$$
h(x) \varphi_{T}(T, x)-\mathcal{L} \varphi(T, x)+\frac{\left|\sigma^{\top}(x) \cdot \nabla_{x} \varphi_{T}(T, x)\right|^{2}}{2 \varphi_{T T}(T, x)}>0
$$

and

$$
\varphi_{T T}(T, x)<0
$$

Now let $\alpha \in L_{l o c}^{2}(W)$ and $\left(\tau_{n}\right)_{n \in \mathbb{N}}$ be a localizing sequence for $\alpha$. In the following we write $M^{\alpha}$ and $X$ instead of $M^{\alpha, T_{0}}$ resp. $X^{x_{0}}$. Define the stopping times

$$
\begin{aligned}
& \theta^{\alpha}=\inf \left\{t \geq 0 \mid\left(M_{t}^{\alpha}-H_{t}, X_{t}\right) \notin \mathcal{N}_{r}\right\} \\
& \theta_{n}^{\alpha}=\theta^{\alpha} \wedge \tau_{n} \wedge n, \quad n \in \mathbb{N} .
\end{aligned}
$$

Applying Itô's formula to $\varphi$ leads to

$$
\begin{aligned}
& U\left(T_{0}, x_{0}\right)=\varphi\left(T_{0}, x_{0}\right) \\
& \quad=E\left[\varphi\left(M_{\theta_{n}^{\alpha}}^{\alpha}-H_{\theta_{n}^{\alpha}}, X_{\theta_{n}^{\alpha}}\right)\right] \\
& \quad+E\left[\int_{0}^{\theta_{n}^{\alpha}}\left(h\left(X_{s}\right) \varphi_{T}-\mathcal{L} \varphi-\frac{\left|\alpha_{s}\right|^{2}}{2} \varphi_{T T}-\left(\nabla_{x} \varphi_{T}\right)^{\top} \cdot \sigma\left(X_{s}\right) \cdot \alpha_{s}\right)\left(M_{s}^{\alpha}-H_{s}, X_{s}\right) d s\right] \\
& \geq E\left[\varphi\left(M_{\theta_{n}^{\alpha}}^{\alpha}-H_{\theta_{n}^{\alpha}}, X_{\theta_{n}^{\alpha}}\right)\right] \\
& \left.\quad+E\left[\int_{0}^{\theta_{n}^{\alpha}}\left(h\left(X_{s}\right) \varphi_{T}-\mathcal{L} \varphi-\sup _{a \in \mathbb{R}^{d}}\left\{\frac{|a|^{2}}{2} \varphi_{T T}-\left(\nabla_{x} \varphi_{T}\right)^{\top} \cdot \sigma\left(X_{s}\right) \cdot a\right)\right\}\right)\left(M_{s}^{\alpha}-H_{s}, X_{s}\right) d s\right] .
\end{aligned}
$$

Here we use that the stochastic integral is a martingale. By (4.6) the previous supremum is given by

$$
-\frac{\left|\sigma^{\top}\left(X_{s}\right) \cdot \nabla_{x} \varphi_{T}\left(M_{s}^{\alpha}-H_{s}, X_{s}\right)\right|^{2}}{2 \varphi_{T T}\left(M_{s}^{\alpha}-H_{s}, X_{s}\right)} .
$$

Therefore, we conclude from (4.5) that

$$
U\left(T_{0}, x_{0}\right) \geq E\left[\varphi\left(M_{\theta_{n}^{\alpha}}^{\alpha}-H_{\theta_{n}^{\alpha}}, X_{\theta_{n}^{\alpha}}\right)\right]
$$

for all $n \in \mathbb{N}$. Since $\varphi$ is bounded on $\overline{\mathcal{N}}_{r}$, taking the limit $n \rightarrow \infty$ results in

$$
\begin{aligned}
U\left(T_{0}, x_{0}\right) & \geq E\left[\varphi\left(M_{\theta^{\alpha}}^{\alpha}-H_{\theta^{\alpha}}, X_{\theta^{\alpha}}\right)\right] \\
& \geq \eta+E\left[U\left(M_{\theta^{\alpha}}^{\alpha}-H_{\theta^{\alpha}}, X_{\theta^{\alpha}}\right)\right],
\end{aligned}
$$


where $\eta:=\min _{(T, x) \in \partial \mathcal{N}_{r}}(\varphi-U)(T, x)>0$. Since $\alpha$ was arbitrary and $\eta>$ 0 is independent of $\alpha$, this contradicts the DPP for $U$. Hence, we have shown that $U(T, x)$ is a subsolution to (4.1) on $A:=\{(T, x) \in(0, \infty) \times$ $\left.\mathbb{R}^{n} \mid U_{T T}(T, x)<0\right\}$. The continuity of $h, U_{T}, \mathcal{L} U$ and $\left|\sigma^{\top} \cdot \nabla_{x} U_{T}\right|^{2} / U_{T T}$ implies that $U$ satisfies

$$
h(x) U_{T}(T, x)-\mathcal{L} U(T, x)+\frac{\left|\sigma^{\top}(x) \cdot \nabla_{x} U_{T}(T, x)\right|^{2}}{U_{T T}(T, x)} \leq 0
$$

for $(T, x) \in \bar{A}$.

Finally, let $\left(T_{0}, x_{0}\right) \in(\bar{A})^{c} \cap(0, \infty) \times\left\{x \in \mathbb{R}^{n} \mid \exists T>0:(T, x) \in \bar{A}\right\}$. Then there exists a neighborhood $\mathcal{N}$ of $\left(T_{0}, x_{0}\right)$ with $\overline{\mathcal{N}} \subseteq(0, \infty) \times \mathbb{R}^{n}$ such that $U_{T T}=0$ on $\overline{\mathcal{N}}$ and $(0, \infty) \times\left\{x_{0}\right\} \cap \overline{\mathcal{N}} \cap \bar{A} \neq \emptyset$. In particular, we have $\nabla_{x} U_{T}=0$ on $\overline{\mathcal{N}}$ by the first part of the proof. Hence, the value function $U$ is linear or constant in $T$ and there exist $c \in \mathbb{R}$ and $g \in \mathcal{C}^{2}\left(\mathbb{R}^{n}\right)$ such that

$$
U(T, x)=c T+g(x), \quad(T, x) \in \overline{\mathcal{N}}
$$

Therefore,

$$
h\left(x_{0}\right) U_{T}\left(T_{0}, x_{0}\right)-\mathcal{L} U\left(T_{0}, x_{0}\right)=\operatorname{ch}\left(x_{0}\right)-\mathcal{L} g\left(x_{0}\right) .
$$

On the other hand there exists $T>0$ such that $\left(T, x_{0}\right) \in \overline{\mathcal{N}} \cap \bar{A}$ with $U\left(T, x_{0}\right)=c T+g\left(x_{0}\right)$ by the continuity of $U$ and

$$
h\left(x_{0}\right) U_{T}\left(T, x_{0}\right)-\mathcal{L} U\left(T, x_{0}\right)=\operatorname{ch}\left(x_{0}\right)-\mathcal{L} g\left(x_{0}\right) \leq 0
$$

by the previous part. Combining (4.7) and (4.8) results in

$$
h\left(x_{0}\right) U_{T}\left(T_{0}, x_{0}\right)-\mathcal{L} U\left(T_{0}, x_{0}\right) \leq 0 .
$$

To sum up, this together with the first part of the Proposition implies that $U$ is a solution to (4.1) on $\bar{A} \cup(0, \infty) \times\left\{x \in \mathbb{R}^{n} \mid \exists T>0:(T, x) \in \bar{A}\right\}$.

\section{Remark 4.2.}

(a) In general $U \in \mathcal{C}^{2}\left((0, \infty) \times \mathbb{R}^{n}\right)$ is not a solution to (4.1) on the set $(\bar{A})^{c} \cap(0, \infty) \times\left\{x \in \mathbb{R}^{n} \mid(T, x) \notin \bar{A}\right.$ for all $\left.T>0\right\}$, see Subsection 7.2 for a counterexample. One can show, however, if there exists an optimal stopping time for any $(T, x)$, then $U$ is a solution to (4.1) on the whole set $\mathbb{R}_{+} \times \mathbb{R}^{n}$.

(b) Let $h$ be continuous. Then the continuity condition in the second part of Proposition 4.1 is necessary: Let $u \in \mathcal{C}^{2}(0, \infty) \times \mathbb{R}^{n}$ be a solution of (4.1) and let $(T, x) \in(0, \infty) \times \mathbb{R}^{n}$. If $u_{T T}(T, x) \neq 0$, then the quotient is continuous in $(T, x)$. Now assume that $u_{T T}(T, x)=0$ and observe that 


$$
\frac{\left|\sigma^{\top}(y) \cdot \nabla_{x} u_{T}(t, y)\right|^{2}}{u_{T T}(t, y)}= \begin{cases}2\left(\mathcal{L} u(t, y)-h(y) u_{T}(t, y)\right), & \text { if } u_{T T}(t, y) \neq 0 \\ 0, & \text { if } u_{T T}(t, y)=0\end{cases}
$$

for all $(t, y) \in(0, \infty) \times \mathbb{R}^{n}$. Thus, for every sequence $\left(T_{n}, x_{n}\right)_{n \in \mathbb{N}} \subseteq$ $(0, \infty) \times \mathbb{R}^{n}$ with $\left(T_{n}, x_{n}\right) \rightarrow(T, x)$ we have

$$
\begin{aligned}
&\left|\frac{\left|\sigma^{\top}\left(x_{n}\right) \cdot \nabla_{x} u_{T}\left(T_{n}, x_{n}\right)\right|^{2}}{u_{T T}\left(T_{n}, x_{n}\right)}\right| \leq 2\left|\mathcal{L} u\left(T_{n}, x_{n}\right)-h\left(x_{n}\right) u_{T}\left(T_{n}, x_{n}\right)\right| \\
& \underset{n \rightarrow \infty}{\longrightarrow} 2\left|\mathcal{L} u(T, x)-h(x) u_{T}(T, x)\right|=0,
\end{aligned}
$$

because $u$ solves $(4.1)$ and $u_{T T}(T, x)=0$. Hence,

$$
\lim _{n \rightarrow \infty} \frac{\left|\sigma^{\top}\left(x_{n}\right) \cdot \nabla_{x} u_{T}\left(T_{n}, x_{n}\right)\right|^{2}}{u_{T T}\left(T_{n}, x_{n}\right)}=0=\frac{\left|\sigma^{\top}(x) \cdot \nabla_{x} u_{T}(T, x)\right|^{2}}{u_{T T}(T, x)}
$$

which implies the continuity of $\left|\sigma^{\top}(x) \cdot \nabla_{x} u_{T}(T, x)\right|^{2} / u_{T T}(T, x)$.

In Example 7.1 the continuity condition is not satisfied and the value function is only a supersolution to (4.1).

Remark 4.3. The convention that $\left|\sigma^{\top}(x) \cdot \nabla_{x} U_{T}(T, x)\right|^{2} / U_{T T}(T, x)=0$ if $U_{T T}(T, x)=0$ is justified by the proof of the first part of Proposition 4.1. There it is shown that

$$
\sup _{a \in \mathbb{R}^{d}}\left\{\frac{|a|^{2}}{2} U_{T T}(T, x)+\left(\nabla_{x} U_{T}(T, x)\right)^{\top} \cdot \sigma(x) \cdot a\right\}= \begin{cases}-\frac{\left|\sigma^{\top}(x) \cdot \nabla_{x} U_{T}(T, x)\right|^{2}}{2 U_{T T}(T, x)}, & \text { if } U_{T T}(T, x)<0, \\ 0, & \text { if } U_{T T}(T, x)=0 .\end{cases}
$$

Remark 4.4. If one replaces Equation (4.1) with

$$
h(x) U_{T}(T, x)-\mathcal{L} U(T, x)-\sup _{a \in \mathbb{R}^{d}}\left\{\frac{|a|^{2}}{2} U_{T T}(T, x)+\left(\nabla_{x} U_{T}(T, x)\right)^{\top} \cdot \sigma(x) \cdot a\right\}=0,
$$

then all the statements of Proposition 4.1 remain true.

Remark 4.5. If we can show that for $X_{t}^{x}=x+W_{t}$ and $h(y)=1, y \in \mathbb{R}^{d}$, then the optimal control $\alpha^{*}$ for $U(T, x)$ is given by $\alpha_{s}^{*}=-2 X_{s}^{x}$, i.e. the optimal stopping time is the first exit time of the ball around 0 with radius $R_{x}$ for some $R_{x}>|x|$ (cf. with Example 2.5). In this case the PDE (4.1) simplifies to

$$
h(x) U_{T}(T, x)-\frac{1}{2} \Delta_{x} U(T, x)+x^{\top} \cdot \nabla_{x} U_{T}(T, x)=0 .
$$




\section{Verification}

In this section we state a classical verification theorem for $U$ and $V$. Recall Remark 4.2 (a): if for all $(T, x)$ there exists an optimal stopping time for problem (1.3), then $U$ is a solution of (4.1) on the whole domain $\mathbb{R}_{+} \times \mathbb{R}^{n}$. Therefore, for a verification it is natural to look for a solution of the PDE (4.1) on the whole set $\mathbb{R}_{+} \times \mathbb{R}^{n}$.

We first show that if $u \in \mathcal{C}^{2}\left((0, \infty) \times \mathbb{R}^{n}\right) \cap \mathcal{C}\left(\left([0, \infty) \times \mathbb{R}^{n}\right)\right.$ is a solution of the PDE (4.1), and some additional mild conditions are satisfied, then $u$ coincides with the value function of the optimal control problem $(\mathcal{U})$. The relation (1.4) allows us then to identify the value function $V$.

\section{Theorem 5.1.}

1. Let $u \in \mathcal{C}^{2}\left((0, \infty) \times \mathbb{R}^{n}\right) \cap \mathcal{C}\left([0, \infty) \times \mathbb{R}^{n}\right)$ be a supersolution of $(4.1)$ which is concave in $T$, satisfies $\left\{(T, x) \mid u_{T T}(T, x)=0\right\} \subseteq\left\{(T, x) \mid \nabla_{x} u_{T}(T, x)=\right.$ $\left.0 \in \mathbb{R}^{n}\right\}, u(0, x)=f(x)$ and has linear growth in $T$ and polynomial growth in $x$. More precisely, there exists $C>0$ and $p \geq 1$ such that

$$
|u(T, x)| \leq C\left(1+T+|x|^{p}\right) .
$$

If $X_{\tau}^{x} \in L^{p}(\Omega)$ for all $\tau \in \mathcal{T}(T)$, then $u(T, x) \geq U(T, x)$.

2. Let $u \in \mathcal{C}^{2}\left((0, \infty) \times \mathbb{R}^{n}\right) \cap \mathcal{C}\left([0, \infty) \times \mathbb{R}^{n}\right)$ be a solution of $(4.1)$ which is concave in $T$ and satisfies $\left.\left\{(T, x) \mid u_{T T}(T, x)\right\}\right\} \subseteq\left\{(T, x) \mid \nabla_{x} u_{T}(T, x)=\right.$ $\left.0 \in \mathbb{R}^{n}\right\}, u(0, x)=f(x)$ and (5.1). Furthermore, assume that

$$
\alpha_{s}^{*}:=-\mathbb{1}_{\left\{u_{T T}<0\right\}}\left(\frac{\left(\nabla_{x} u_{T}\right)^{\top} \cdot \sigma}{u_{T T}}\right)\left(M_{s}^{*}-H_{s}, X_{s}^{x}\right) \in L_{l o c}^{2}(W),
$$

where

$d M_{s}^{*}=-\mathbb{1}_{\left\{M_{s}^{*}>H_{s}\right\}}\left(\mathbb{1}_{\left\{u_{T T}<0\right\}} \frac{\left(\nabla_{x} u_{T}\right)^{\top} \cdot \sigma}{u_{T T}}\right)\left(M_{s}^{*}-H_{s}, X_{s}^{x}\right) \cdot d W_{s}, \quad M_{0}^{*}=T$.

If $X_{\tau}^{x} \in L^{p}(\Omega)$ for all $\tau \in \mathcal{T}(T)$, then $u(T, x)=U(T, x)$ and $\left(\alpha_{s}^{*}\right)$ is an optimal control.

The corresponding optimal stopping time $\tau^{*}$ in (1.2) is given by

$$
\tau^{*}=\left\{t \geq 0 \mid M_{t}^{*} \leq H_{t}\right\}
$$

3. If the assumptions in 2. are satisfied for all $s \leq T$, then

$$
V(T, x)=\sup _{s \in[0, T]} u(s, x)=u(T \wedge \widetilde{T}(x), x),
$$

where $\widetilde{T}(x)=\inf \left\{t \geq 0 \mid u_{T}(t, x) \leq 0\right\}$; and an optimal control $\left(\alpha_{s}^{*}, m^{*}\right)$ for $V(T, x)$ is given by

$$
\alpha_{s}^{*}=-\mathbb{1}_{\left\{u_{T T}<0\right\}}\left(\frac{\left(\nabla_{x} u_{T}\right)^{\top} \cdot \sigma}{u_{T T}}\right)\left(M_{s}^{*}-H_{s}, X_{s}^{x}\right), \quad m^{*}=T \wedge \widetilde{T}(x),
$$


where

$d M_{s}^{*}=-\mathbb{1}_{\left\{M_{s}^{*}>H_{s}\right\}}\left(\mathbb{1}_{\left\{u_{T T}<0\right\}} \frac{\left(\nabla_{x} u_{T}\right)^{\top} \cdot \sigma}{u_{T T}}\right)\left(M_{s}^{*}-H_{s}, X_{s}^{x}\right) \cdot d W_{s}, \quad M_{0}^{*}=m^{*}$.

The corresponding optimal stopping time $\tau^{*}$ in (1.2) is given by

$$
\tau^{*}=\inf \left\{t \geq 0 \mid M_{t}^{*} \leq H_{t}\right\} .
$$

Proof. 1. We first show that every concave supersolution $u$ of (4.1) with the same initial condition $u(0, T)=f(x)$ satisfying the assumptions given in the proposition dominates $U$. Let $(T, x) \in(0, \infty) \times \mathbb{R}^{n}$. For $\left(\alpha_{s}\right) \in L_{l o c}^{2}(W)$ let $\left(M_{s}^{\alpha, T}\right)$ be the unique strong solution of $(2.2)$ and let $\left(\tau_{n}\right)_{n \in \mathbb{N}}$ be a localizing sequence for $\alpha$. For every $n \in \mathbb{N}$ define the stopping times

$$
\begin{aligned}
& \rho_{n}=\inf \left\{t \geq 0 \mid M_{t}^{\alpha, T}-H_{t} \leq \frac{1}{n}\right\} \\
& \sigma_{n}=\inf \left\{t \geq 0 \mid M_{t}^{\alpha, T}-H_{t} \geq n \text { or }\left|X_{t}^{x}\right| \geq n\right\}
\end{aligned}
$$

and $\theta_{n}=\rho_{n} \wedge \sigma_{n} \wedge \tau_{n} \wedge n$. For $n$ sufficiently large, Itô's formula implies

$$
\begin{aligned}
& u\left(M_{\theta_{n}}^{\alpha, T}-H_{\theta_{n}}, X_{\theta_{n}}^{x}\right)-u(T, x) \\
& =\int_{0}^{\theta_{n}}\left(u_{T}\left(M_{s}^{\alpha, T}-H_{s}, X_{s}^{x}\right) \alpha_{s}+\left(\nabla_{x} u\left(M_{s}^{\alpha, T}-H_{s}, X_{s}^{x}\right)\right)^{\top} \cdot \sigma\left(X_{s}^{x}\right)\right) \cdot d W_{s} \\
& \quad+\int_{0}^{\theta_{n}}\left(-h\left(X_{s}^{x}\right) u_{T}+\mathcal{L} u+\frac{\left|\alpha_{s}\right|^{2}}{2} u_{T T}+\left(\nabla_{x} u_{T}\right)^{\top} \cdot \sigma\left(X_{s}^{x}\right) \cdot \alpha_{s}\right)\left(M_{s}^{\alpha, T}-H_{s}, X_{s}^{x}\right) d s .
\end{aligned}
$$

Hence,

$$
\begin{aligned}
u(T, x) \geq & E\left[u\left(M_{\theta_{n}}^{\alpha, T}-H_{\theta_{n}}, X_{\theta_{n}}^{x}\right)\right]-E\left[\int_{0}^{\theta_{n}}\left(u_{T} \alpha_{s}+\left(\nabla_{x} u\right)^{\top} \cdot \sigma\left(X_{s}^{x}\right)\right)\left(M_{s}^{\alpha, T}-H_{s}, X_{s}^{x}\right) d W_{s}\right] \\
& +E\left[\int_{0}^{\theta_{n}}\left(h\left(X_{s}^{x}\right) u_{T}-\mathcal{L} u-\frac{\left|\alpha_{s}\right|^{2}}{2} u_{T T}-\left(\nabla_{x} u_{T}\right)^{\top} \cdot \sigma \cdot \alpha_{s}\right)\left(M_{s}^{\alpha, T}-H_{s}, X_{s}^{x}\right) d s\right] \\
\geq & E\left[u\left(M_{\theta_{n}}^{\alpha, T}-H_{\theta_{n}}, X_{\theta_{n}}^{x}\right)\right]-E\left[\int_{0}^{\theta_{n}}\left(u_{T} \alpha_{s}+\left(\nabla_{x} u\right)^{\top} \cdot \sigma\left(X_{s}^{x}\right)\right)\left(M_{s}^{\alpha, T}-H_{s}, X_{s}^{x}\right) d W_{s}\right] \\
& +E\left[\int_{0}^{\theta_{n}}\left(h\left(X_{s}^{x}\right) u_{T}-\mathcal{L} u-\sup _{a \in \mathbb{R}^{d}}\left\{\frac{|a|^{2}}{2} u_{T T}-\left(\nabla_{x} u_{T}\right)^{\top} \cdot \sigma \cdot a\right\}\right)\left(M_{s}^{\alpha, T}-H_{s}, X_{s}^{x}\right) d s\right]
\end{aligned}
$$

With the same arguments as in the proof of Proposition 4.1 it follows that the stochastic integral is a true martingale and its expectation vanishes. Since $u$ is a concave supersolution of (4.1) with $\left\{(T, x) \mid u_{T T}(T, x)=0\right\} \subseteq$ $\left\{(T, x) \mid \nabla_{x} u_{T}(T, x)=0 \in \mathbb{R}^{n}\right\}$, we have

$$
u(T, x) \geq E\left[u\left(M_{\theta_{n}}^{\alpha, T}-H_{\theta_{n}}, X_{\theta_{n}}^{x}\right)\right] .
$$


For $n \rightarrow \infty, \theta_{n}$ converges to $\tau:=\inf \left\{t \geq 0 \mid M_{t}^{\alpha, T} \leq H_{t}\right\}$. The pathwise continuity of $X_{s}^{x}$ and $M_{s}^{\alpha, T}-H_{s}$ imply that

$M_{\theta_{n}}^{\alpha, T}-H_{\theta_{n}} \underset{n \rightarrow \infty}{\longrightarrow} M_{\tau}^{\alpha, T}-H_{\tau}=0 \quad$ and $\quad X_{\theta_{n}}^{x} \underset{n \rightarrow \infty}{\longrightarrow} X_{\tau}^{x}=X_{G\left(M_{\tau}^{\alpha, T}\right)}^{x}=X_{G\left(M_{\infty}^{\alpha, T}\right)}^{x}, \quad$ a.s.

We deduce that

$$
u\left(M_{\theta_{n}}^{\alpha, T}-H_{\theta_{n}}, X_{\theta_{n}}^{x}\right) \underset{n \rightarrow \infty}{\longrightarrow} u\left(0, X_{G\left(M_{\infty}^{\alpha, T}\right)}^{x}\right)=f\left(X_{G\left(M_{\infty}^{\alpha, T}\right)}^{x}\right)
$$

from the continuity of $u$ on $[0, \infty) \times \mathbb{R}^{n}$ and the initial condition. (5.1) implies that

$\left|u\left(M_{\theta_{n}}^{\alpha, T}-H_{\theta_{n}}, X_{\theta_{n}}^{x}\right)\right| \leq C\left(1+M_{\theta_{n}}^{\alpha, T}-H_{\theta_{n}}+\left|X_{\theta_{n}}^{x}\right|^{p}\right) \leq C\left(1+M_{\theta_{n}}^{\alpha, T}+\left|X_{\theta_{n}}^{x}\right|^{p}\right) \in L^{1}(\Omega)$,

because $\left|X_{\theta_{n}}^{x}\right| \leq\left|X_{\tau}^{x}\right| \in L^{p}(\Omega)$. Then it follows from the dominated convergence theorem that for all $\left(\alpha_{s}\right) \in L_{l o c}^{2}(W)$

$$
u(T, x) \geq E\left[f\left(X_{G\left(M_{\infty}^{\alpha, T}\right)}^{x}\right)\right]
$$

and hence, $u(T, x) \geq U(T, x)$.

2. Now assume that for $(T, x) \in(0, \infty) \times \mathbb{R}^{n}$

$$
\alpha_{s}^{*}:=-\left(\mathbb{1}_{\left\{u_{T T}<0\right\}} \frac{\left(\nabla_{x} u_{T}\right)^{\top} \cdot \sigma}{u_{T T}}\right)\left(M_{s}^{*}-H_{s}, X_{s}^{x}\right) \in L_{l o c}^{2}(W),
$$

where

$d M_{s}^{*}=-\mathbb{1}_{\left\{M_{s}^{*}>H_{s}\right\}}\left(\mathbb{1}_{\left\{u_{T T}<0\right\}} \frac{\left(\nabla_{x} u_{T}\right)^{\top} \cdot \sigma}{u_{T T}}\right)\left(M_{s}^{*}-H_{s}, X_{s}^{x}\right) \cdot d W_{s}, \quad M_{0}^{*}=T$.

Let $\left(\tau_{n}\right)_{n \in \mathbb{N}}$ be a localizing sequence for $\alpha^{*}$ and define the stopping times $\rho_{n}, \sigma_{n}$ and $\theta_{n}$ as in the first part of the proof and apply Itô's formula to $u\left(M_{\theta_{n}}^{*}-H_{\theta_{n}}, X_{\theta_{n}}^{x}\right)$. Notice that the definition of $\alpha^{*}$ implies that

$$
u(T, x)=E\left[u\left(M_{\theta_{n}}^{*}-H_{\theta_{n}}, X_{\theta_{n}}^{x}\right)\right] .
$$

Finally, we have

$$
\left|u\left(M_{\theta_{n}}^{*}-H_{\theta_{n}}, X_{\theta_{n}}^{x}\right)\right| \leq C\left(1+M_{\theta_{n}}^{*}-H_{\theta_{n}}+\left|X_{\theta_{n}}^{x}\right|^{p}\right) \leq C\left(1+M_{\theta_{n}}^{*}+\left|X_{\tau}^{x}\right|^{p}\right) \in L^{1}(\Omega)
$$

Therefore, taking the limit $n \rightarrow \infty$ results in

$$
u(T, x)=E\left[f\left(X_{G\left(M_{\infty}^{*}\right)}^{x}\right)\right] \leq U(T, x),
$$

which implies the second claim.

3. For the last part notice that $u_{T}$ is non-increasing in $T$ for fixed $x$ by the concavity of $u$ in $T$. Thus, $m^{*}=\widetilde{T}(x) \wedge T$ and the corresponding optimal $\alpha^{*}$ given in 2. are optimal for $V(T, x)$. 
Remark 5.2. Observe that Brownian motion $X^{x}$ starting in $x \in \mathbb{R}^{d}$ satisfies $X_{\tau}^{x} \in L^{2}(\Omega)$ for all $\tau \in \mathcal{T}(T)$, because $E\left[\left|X_{\tau}^{x}\right|^{2}\right]=|x|^{2}+d E[\tau]=|x|^{2}+d T$.

Corollary 5.3. If the assumptions of Theorem 5.1 are satisfied, then $V$ is a solution to

$$
\min \left\{V_{T}(T, x), h(x) V_{T}(T, x)-\mathcal{L} V(T, x)+\frac{\left|\sigma^{\top}(x) \cdot \nabla_{x} V_{T}(T, x)\right|^{2}}{2 V_{T T}(T, x)}\right\}=0
$$

on $(0, \infty) \times \mathbb{R}^{n} \backslash \partial\left\{(T, x) \in(0, \infty) \times \mathbb{R}^{n} \mid V_{T}(T, x)=0\right\}$ with initial condition $V(0, x)=f(x)$, where $\partial D$ denotes the boundary of an arbitrary set $D \subseteq$ $(0, \infty) \times \mathbb{R}^{n}$

We next apply Theorem 5.1 in order to determine the optimal stopping time for various examples.

Example 5.4 (Maximizing the Euclidean norm of a Brownian motion). Let $f(y)=|y|, h(y)=1, y \in \mathbb{R}^{d}$, and $X_{t}^{x}=x+W_{t}, t \geq 0$, be a $d$-dimensional Brownian motion starting in $x \in \mathbb{R}^{d}$. Then the value function $V$ of the optimal control problem $(\mathcal{V})$ is given by

$$
V(T, x):=\sqrt{d T+|x|^{2}} .
$$

An optimal control for $V(T, x)$ is $\left(\alpha_{s}^{*}, m^{*}\right)=\left(-2 X_{s}^{x}, T\right)$ and the corresponding optimal stopping time is the first exit time of the ball around 0 with radius $\sqrt{d T+|x|^{2}}$.

To see this, we first show that $u(T, x):=\sqrt{d T+|x|^{2}}$ is the value function of the optimal control problem $(\mathcal{U})$ using Theorem 5.1.

It is obvious that $u \in \mathcal{C}^{2}\left((0, \infty) \times \mathbb{R}^{d}\right) \cap \mathcal{C}\left([0, \infty) \times \mathbb{R}^{d}\right), u(0, x)=|x|=f(x)$ and that $T \mapsto \sqrt{d T+|x|^{2}}$ is concave for fixed $x \in \mathbb{R}^{d}$. Moreover, $u_{T T}<0$ on $(0, \infty) \times \mathbb{R}^{d}$,

$$
|u(T, x)| \leq \sqrt{d T}+|x| \leq d(1+T+|x|)
$$

and $u$ satisfies the PDE (4.1):

$$
\begin{aligned}
& h(x) u_{T}(T, x)-\frac{1}{2} \Delta_{x} u(T, x)+\frac{\left|\nabla_{x} u_{T}(T, x)\right|^{2}}{2 u_{T T}(T, x)} \\
& =\frac{d}{2\left(d T+|x|^{2}\right)^{\frac{1}{2}}}-\frac{1}{2} \sum_{i=1}^{d}\left(\frac{1}{\left(d T+|x|^{2}\right)^{\frac{1}{2}}}-\frac{x_{i}^{2}}{\left(d T+|x|^{2}\right)^{\frac{3}{2}}}\right)-\frac{1}{2 d^{2}} \sum_{i=1}^{d} \frac{d^{2} x_{i}^{2}}{\left(d T+|x|^{2}\right)^{\frac{3}{2}}} \\
& \quad=0 .
\end{aligned}
$$

Define

$$
\alpha_{s}^{*}=-\left(\frac{\nabla_{x} u_{T}}{u_{T T}}\right)\left(M_{s}^{*}-s, X_{s}^{x}\right)=-\frac{2 X_{s}^{x}}{d}, \quad M_{t}^{*}=T+\int_{0}^{t} \alpha_{s}^{*} \mathbb{1}_{\left\{M_{s}^{*}>s\right\}} d W_{s} .
$$


Then, $\left(\alpha_{s}^{*}\right)_{s \geq 0} \in L_{l o c}^{2}(W)$ and

$$
M_{t}^{*}=T+|x|^{2}-\left|X_{t \wedge \sigma}^{x}\right|^{2}+t \wedge \sigma
$$

where $\sigma=\inf \left\{t \geq 0 \mid M_{t}^{*} \leq t\right\}=\inf \left\{t \geq 0 \mid X_{t}^{x} \notin\left(-\sqrt{d T+|x|^{2}}, \sqrt{d T+|x|^{2}}\right)\right\}$. Since $X_{\tau}^{x} \in L^{2}(\Omega)$ for all $\tau \in \mathcal{T}(T)$ by Remark 5.2, the verification theorem shows that $u(T, x)$ is the value function of the optimal control problem $(\mathcal{U})$. Moreover, $u_{T}>0$ implies that $V(T, x)=U(T, x)$ with optimal control $\left(\alpha^{*}, T\right)$. Using the one-to-one correspondence established in Proposition 2.3, the optimal stopping time in (1.2) is given by $\sigma$.

We remark that in this example one can perform a verification without using Theorem 5.1: Observe that

$$
E\left[\left|X_{\tau}^{x}\right|\right] \leq E\left[\left|X_{\tau}^{x}\right|^{2}\right]^{\frac{1}{2}}=\sqrt{d T+|x|^{2}}
$$

by the Cauchy-Schwarz inequality and the fact that $E\left[\left|X_{\tau}^{x}\right|^{2}\right]=x^{2}+d T$ for all stopping times $\tau \in \mathcal{T}(T)$. Hence, $V(T, x) \leq \sqrt{d T+|x|^{2}}$. Notice that the first exit time $\tau(T, x)$ of a ball around 0 with radius $\sqrt{d T+|x|^{2}}$ has expectation $T$, see Chapter 4.2.E in [6] and, thus,

$$
V(T, x) \geq E\left[\left|X_{\tau(T, x)}^{x}\right|\right]=\sqrt{d T+|x|^{2}},
$$

which shows equality.

Example 5.5 (A value function which is strictly decreasing in time). Here we present an example, where the value function $U$ is a classical solution to (4.1), but $V$ is not in $\mathcal{C}^{2}((0, \infty) \times \mathbb{R})$.

Consider $f(y)=-y^{4}+y^{2}, h(y)=1, y \in \mathbb{R}$, and let $X_{t}^{x}=x+W_{t}, t \geq 0$, be a one-dimensional Brownian motion starting in $x \in \mathbb{R}$. We will show that

$$
u(T, x):=-x^{4}+x^{2}+T-T^{2}-2 x^{2} T
$$

is the value function of $(\mathcal{U})$ with optimal control $\alpha_{s}^{*}=-2 X_{s}^{x}, s \geq 0$, whereas the value function $V$ of the optimal control problem $(\mathcal{V})$ is given by

$$
V(T, x)= \begin{cases}\frac{1}{4}, & \text { if } x^{2} \leq \frac{1}{2}, T \geq \frac{1}{2}-x^{2} \\ -x^{4}+x^{2}+T-T^{2}-2 x^{2} T, & \text { if } x^{2} \leq \frac{1}{2}, T<\frac{1}{2}-x^{2} \\ -x^{4}+x^{2}, & \text { if } x^{2}>\frac{1}{2}\end{cases}
$$

The optimal control is given by $\left(\alpha^{*}, T^{*}\right)$, where $T^{*}=\left(\left(1 / 2-x^{2}\right) \wedge T\right)^{+}$.

The function $u$ is in $\mathcal{C}^{2}((0, \infty) \times \mathbb{R}) \cap \mathcal{C}([0, \infty) \times \mathbb{R}), u(0, x)=-x^{4}+$ $x^{2}=f(x), u$ is concave in $T$ with $u_{T T}=-2<0$ on $(0, \infty) \times \mathbb{R}$ and $u$ satisfies the PDE (4.1). Notice that $u$ does not satisfy the growth condition (5.1), but nevertheless we can show that $E\left[u\left(M_{\theta_{n}}^{\alpha, T}-\theta_{n}, X_{\theta_{n}}^{x}\right)\right]$ converges to 
$E\left[f\left(X_{M_{\infty}^{\alpha, T}}^{x}\right)\right]$ for all square integrable stopping times $\tau \in \mathcal{T}(T)$ and that $E\left[f\left(X_{\tau}^{x}\right)\right]=-\infty$ if $\tau$ is not square integrable. Thus, we conclude that

$$
u(T, x) \geq \sup _{\tau \in \mathcal{T}(T) \text { with } E\left[\tau^{2}\right]<\infty} E\left[f\left(X_{\tau}^{x}\right)\right]=U(T, x) .
$$

Furthermore, we have $u(T, x)=E\left[f\left(X_{\tau^{*}}^{x}\right)\right]$, where $\tau^{*}$ denotes the first exit time of $\left(-\sqrt{T+x^{2}}, \sqrt{T+x^{2}}\right)$. Note that $E\left[\left(\tau^{*}\right)^{2}\right]=5 / 3\left(T+x^{2}\right)^{2}$ by [4], Theorem 8.5.9. Therefore, $u$ is the value function of the optimal control problem $(\mathcal{U})$. By Theorem 5.1 the value function $V$ is given by $(5.3)$ and thus is not in $\mathcal{C}^{2}((0, \infty) \times \mathbb{R})$.

Example 5.6 (The optimal stopping time is not a first hitting time of two points). In the previous two examples the optimal stopping times are first exit times of balls, i.e. the optimal control is of the form $\alpha_{t}^{*}=-2 X_{t} / d$. We now present an example where this is not the case.

For $f(y)=y^{2} \mathbb{1}_{\{|y| \geq 1\}}, h(y)=1, y \in \mathbb{R}$, and a one-dimensional Brownian motion $X_{t}^{x}=x+W_{t}, t \geq 0$, which starts in $x \in \mathbb{R}$, the value function $U$ is given by

$$
u(T, x)= \begin{cases}\frac{T}{T+(1-|x|)^{2}}, & T<|x|(1-|x|), \\ T+x^{2}, & T \geq|x|(1-|x|) .\end{cases}
$$

Note that $u$ is only in $\mathcal{C}^{1,1}((0, \infty) \times \mathbb{R})$. Therefore we slightly modify the arguments used in the proof of Theorem 5.1 to show that $u$ is indeed the value function $U$ of the optimal control problem.

Let $\mathcal{R}=\{(t, x) \in(0, \infty) \times \mathbb{R}|t<| x \mid(1-|x|)\}$. In order to verify that $U=u$ on $\mathcal{R}$, let $(T, x) \in \mathcal{R}, \alpha \in L_{\text {loc }}^{2}(W)$ and define the stopping times

$$
\begin{gathered}
\sigma=\inf \left\{t \geq 0 \mid\left(M_{t}^{\alpha, T}-t, X_{t}^{x}\right) \notin \mathcal{R}\right\} \\
\theta_{n}=\inf \left\{t \geq 0 \mid M_{t}^{\alpha, T}-t \leq \frac{1}{n}\right\} \wedge \tau_{n}
\end{gathered}
$$

where $\left(\tau_{n}\right)_{n \in \mathbb{N}}$ is a localizing sequence for $\alpha$. Observe that $u \in \mathcal{C}^{2}(\mathcal{R})$ and that the stochastic integral is a martingale on $\left[0, \theta_{n} \wedge \sigma\right]$. Hence,

$$
\begin{aligned}
u(T, x)= & E\left[u\left(M_{\theta_{n} \wedge \sigma}^{\alpha, T}-\theta_{n} \wedge \sigma, X_{\theta_{n} \wedge \sigma}^{x}\right)\right] \\
& +E\left[\int_{0}^{\theta_{n} \wedge \sigma}\left(u_{T}-\mathcal{L} u-\frac{\left|\alpha_{s}\right|^{2}}{2} u_{T T}-\alpha_{s} u_{T x}\right)\left(M_{s}^{\alpha, T}-s, X_{s}^{x}\right) d s\right] \\
\geq & E\left[u\left(M_{\theta_{n} \wedge \sigma}^{\alpha, T}-\theta_{n} \wedge \sigma, X_{\theta_{n} \wedge \sigma}^{x}\right)+\int_{0}^{\theta_{n} \wedge \sigma}\left(u_{T}-\mathcal{L} u+\frac{1}{2} \frac{u_{T x}^{2}}{u_{T T}}\right)\left(M_{s}^{\alpha, T}-s, X_{s}^{x}\right) d s\right] \\
= & E\left[u\left(M_{\theta_{n} \wedge \sigma}^{\alpha, T}-\theta_{n} \wedge \sigma, X_{\theta_{n} \wedge \sigma}^{x}\right)\right] .
\end{aligned}
$$


In the last step we use that $u$ is a solution to (4.1) on $\mathcal{R}$. The stopping times $\theta_{n}$ converges to $\tau$ for $n \rightarrow \infty$. Therefore,

$$
\begin{aligned}
u(T, x) & \geq E\left[u\left(M_{\tau \wedge \sigma}^{\alpha, T}-\tau \wedge \sigma, X_{\tau \wedge \sigma}^{x}\right)\right] \\
& =E\left[\mathbb{1}_{\{\tau \leq \sigma\}} u\left(M_{\tau}^{\alpha, T}-\tau, X_{\tau}^{x}\right)+\mathbb{1}_{\{\sigma<\tau\}} u\left(M_{\sigma}^{\alpha, T}-\sigma, X_{\sigma}^{x}\right)\right] \\
& =E\left[\mathbb{1}_{\{\tau \leq \sigma\}} f\left(X_{\tau}^{x}\right)+\mathbb{1}_{\{\sigma<\tau\}}\left(M_{\sigma}^{\alpha, T}-\sigma+\left(X_{\sigma}^{x}\right)^{2}\right)\right] .
\end{aligned}
$$

Conditioning on $\mathcal{F}_{\sigma}$ and applying Itô's formula once more, we obtain

$$
u(T, x) \geq E\left[f\left(X_{\tau}^{x}\right)\right]
$$

and hence we conclude that $u(T, x) \geq U(T, x)$. The stopping time $\tau(T, x)=$ $\inf \left\{t \geq 0 \mid X_{t}^{x} \notin(x-T /(1-x), 1)\right\}$ if $x>0$ resp. $\tau(T, x)=\inf \left\{t \geq 0 \mid X_{t}^{x} \notin\right.$ $(-1, x+T /(1+x)\}$ if $x<0$ gives

$$
u(T, x)=E\left[f\left(X_{\tau(T, x)}^{x}\right)\right] \leq U(T, x) .
$$

Hence, $u=U$ on $\mathcal{R}$. For $(T, x) \in \mathcal{R}^{c}$, note that $f(y) \leq y^{2}$ and, thus,

$$
U(T, x) \leq \sup _{\tau \in \mathcal{T}(T)} E\left[\left(X_{\tau}^{x}\right)^{2}\right]=x^{2}+T=u(T, x)
$$

For the reverse inequality let $\vartheta=\inf \left\{t \geq 0 \mid\left(T-t, X_{t}^{x}\right) \in \mathcal{R}\right\}$ and define

$$
\alpha_{s}^{*}=\left(-2 X_{s}^{x}+\operatorname{sign}\left(X_{\vartheta}^{x}\right)\right) \mathbb{1}_{\{s \geq \vartheta\}} .
$$

Then $\alpha^{*} \in L_{l o c}^{2}(W)$. Using the arguments given in the proof of the second part of Theorem 5.1, we obtain $u(T, x)=E\left[f\left(X_{M_{\infty}^{*}}^{*}\right)\right] \leq U(T, x)$.

Notice that, for $T>|x|(1-|x|)$, the optimal strategy can be described by the words: "Do nothing until $\vartheta \wedge T$; after $\vartheta$, provided $\vartheta<T$, control the process $M$ in such a way that the space-time process $\left(X_{s}^{x}, M_{s}-s\right)$ stays on the graph of $x(1-x)$ if $X_{\vartheta}^{x}>0$ resp. $-x(1+x)$ if $X_{\vartheta}^{x}<0$." Figure 1 illustrates possible paths the pair $\left(X_{t}^{0}, M_{t}^{*, 1}-t\right)$ can take.

Example 5.7 (Constraint on the time where a Brownian motion stays in $(0, \infty))$. In this example we allow for stopping times such that the expected time, which a Brownian motion spends above 0 until $\tau$, equals $T$. Moreover, the payoff is only greater than 0 if the argument is positive, otherwise it equals 0 .

Consider a one-dimensional Brownian motion $X_{t}^{x}, t \geq 0$, which starts in $x \in \mathbb{R}$. Let $h(x)=\mathbb{1}_{\{x \geq 0\}}$ be the constraint function and $f(x)=x^{2} \mathbb{1}_{\{x \geq 0\}}$ be the payoff function. Note that Assumption (A) is satisfied. The value function $U$ is given by

$$
U(T, x)=T+x^{2} \mathbb{1}_{\{x \geq 0\}}=T+f(x)
$$




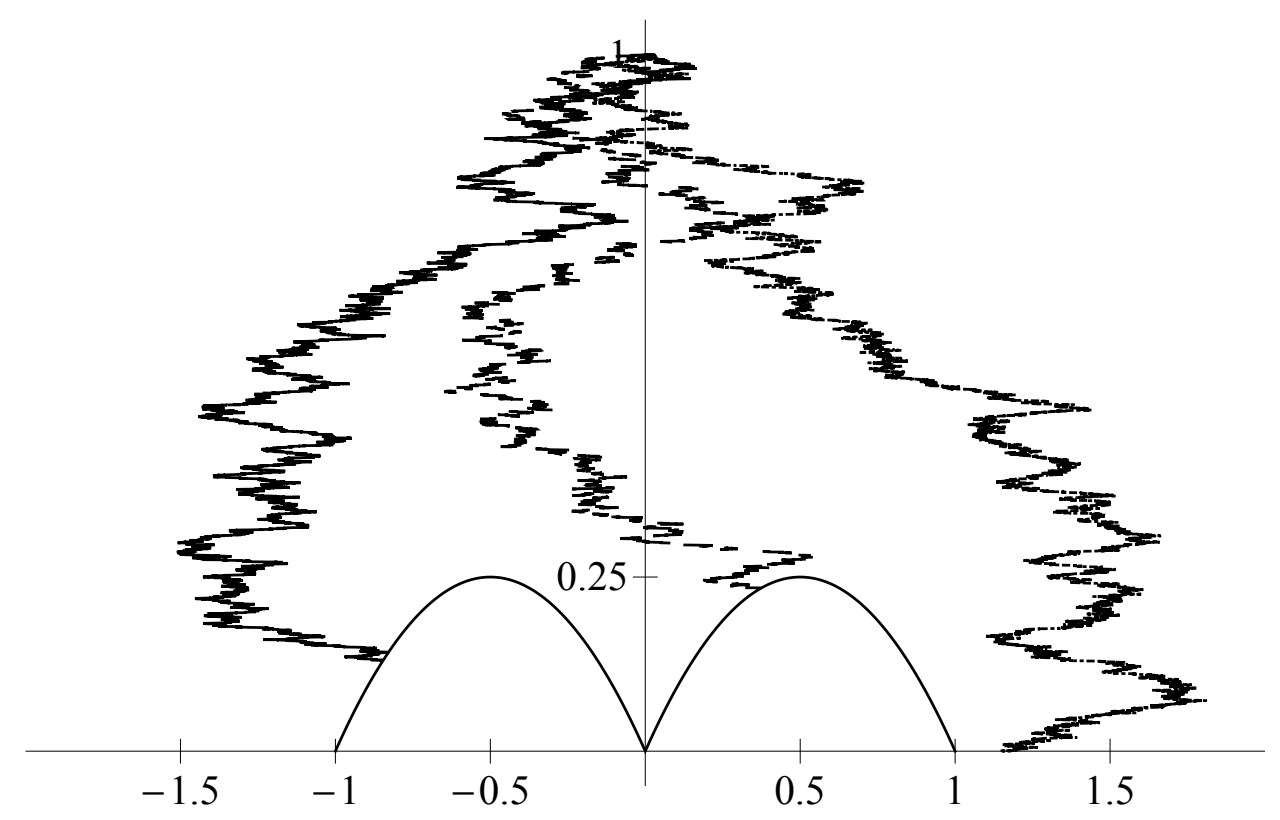

Figure 1: The figure depicts three realizations of the pair $\left(X_{t}^{0}, M_{t}^{*, 1}-t\right)$ in Example 5.6. The $y$-axis is the conditional expected remaining time.

First, notice that $f$ is not in $\mathcal{C}^{2}(\mathbb{R})$ and hence we cannot directly use the verification theorem. Nevertheless, since the first derivative of $f$ is absolutely continuous, we apply an Itô-formula for such functions, see [6], Chapter 3.7, Problem 7.3, and then continue as in the proof of Theorem 5.1. Let $\alpha \in L_{l o c}^{2}$ and $\left(\tau_{n}\right)_{n \in \mathbb{N}}$ be a localizing sequence for $\alpha$. For every $n \in \mathbb{N}$ define the stopping times

$$
\begin{aligned}
& \rho_{n}=\inf \left\{t \geq 0 \mid M_{t}^{\alpha, T}-H_{t} \leq \frac{1}{n}\right\} \\
& \sigma_{n}=\inf \left\{t \geq 0 \mid M_{t}^{\alpha, T}-H_{t} \geq n \text { or }\left|X_{t}^{x}\right| \geq n\right\}
\end{aligned}
$$

and $\theta_{n}=\rho_{n} \wedge \sigma_{n} \wedge \tau_{n} \wedge n$. Then we have

$$
\begin{aligned}
\left.U\left(M_{\theta_{n}}^{\alpha, T}-H_{\theta_{n}}, X_{\theta_{n}}^{x}\right)\right] & =E\left[M_{\theta_{n}}^{\alpha, T}-H_{\theta_{n}}+f\left(X_{\theta_{n}}^{x}\right)\right] \\
& =T+f(x)+E\left[\int_{0}^{\theta_{n}} \frac{1}{2} f^{\prime \prime}\left(X_{s}^{x}\right)-h\left(X_{s}^{x}\right) d s\right]+E\left[\int_{0}^{\theta_{n}} f^{\prime}\left(X_{s}^{x}\right) d W_{s}\right] \\
& =T+f(x) \\
& =U(T, x),
\end{aligned}
$$

because the stochastic integral is a martingale and $f^{\prime \prime}(x)=2 h(x), \lambda$-almost everywhere. For $n \rightarrow \infty, \theta_{n}$ converges to $\tau:=\inf \left\{t \geq 0 \mid M_{t}^{\alpha, T} \leq H_{t}\right\}$. Since 
$U$ is continuous, the pathwise continuity of $X_{s}^{x}$ and $M_{s}^{\alpha, T}-H_{s}$ imply that

$$
U\left(M_{\theta_{n}}^{\alpha, T}-H_{\theta_{n}}, X_{\theta_{n}}^{x}\right) \underset{n \rightarrow \infty}{\longrightarrow} U\left(0, X_{G\left(M_{\infty}^{\alpha, T}\right)}^{x}\right)=f\left(X_{G\left(M_{\infty}^{\alpha, T}\right)}^{x}\right)
$$

and therefore

$$
U(T, x)=\sup _{\alpha \in L_{l o c}^{2}} E\left[f\left(X_{G^{x}\left(M_{\infty}^{\alpha, T}\right)}^{x}\right)\right] .
$$

For $x>0$ every stopping time $\tau$ with mean $T$ and such that $X_{\tau}^{x} \in[0, \infty)$ is optimal. For $x \leq 0$ an optimal stopping time is given by

$$
\tau=\inf \left\{t \geq 0 \mid X_{t}^{x}=\sqrt{T}\right\}
$$

To see this, notice that

$$
E\left[H_{\tau}^{x}\right]=E\left[\int_{0}^{\tau} h\left(X_{s}^{x}\right) d s\right]=E\left[\int_{0}^{\tau} \mathbb{1}_{\left\{X_{s}^{x} \in[0, \infty)\right\}} d s\right]=E\left[\int_{0}^{\infty} 2 L_{\tau}^{x}(y) d y\right]
$$

by the occupation time formula, where $\left(L_{s}^{x}(y)\right)_{s \geq 0}$ denotes the local time of $X^{x}$ in $y$. Using Tanaka's formula gives

$$
E\left[H_{\tau}^{x}\right]=2 \int_{0}^{\infty} E\left[\left(X_{\tau}^{x}-y\right)^{+}-(x-y)^{+}\right] d y=2 \int_{0}^{\sqrt{T}} \sqrt{T}-y d y=T .
$$

And we obtain

$$
E\left[f\left(X_{\tau}^{x}\right)\right]=T
$$

Since $U$ is strictly increasing in $T$ the value functions $U$ and $V$ coincide.

\section{The Lagrangian Dual Problem}

In this section we briefly compare our solution method with a Lagrange approach for solving the stopping problem (1.2).

We first show the concave conjugate of $V(T, x)$, considered as a function in $T$, is the value function of an unconstrained stopping problem with infinite time horizon. To this end we define $\mathcal{T}=\bigcup_{T \geq 0} \mathcal{T}(T)$.

Proposition 6.1 (cf. [7]). Let $w: \mathbb{R}_{+} \times \mathbb{R}^{d} \rightarrow \mathbb{R} \cup\{+\infty\}$ denote the function

$$
w(\lambda, x)=\sup _{\tau \in \mathcal{T}} E\left[f\left(X_{\tau}^{x}\right)-\lambda H_{\tau}\right]
$$

and let $V^{*}: \mathbb{R}_{+} \times \mathbb{R}^{d} \rightarrow \mathbb{R} \cup\{-\infty\}$ be the conjugate function of $V$ with respect to the first argument

$$
V^{*}(\lambda, x)=\inf _{T \geq 0}(T \lambda-V(T, x)) .
$$

Then it holds that $V^{*}=-w$. 
Proof. We have for all $(\lambda, x) \in \mathbb{R}_{+} \times \mathbb{R}^{d}$

$$
-V^{*}(\lambda, x)=\sup _{T \geq 0}(V(T, x)-T \lambda) \leq \sup _{T \geq 0} \sup _{\tau \in \mathcal{S}(T)} E\left[f\left(X_{\tau}^{x}\right)-\lambda H_{\tau}\right]=w(\lambda, x) .
$$

Moreover, for all $x \in \mathbb{R}^{d}$ and stopping times $\tau \in \mathcal{T}$ it holds that $E\left[f\left(X_{\tau}^{x}\right)\right] \leq$ $V\left(E\left[H_{\tau}\right], x\right)$. This implies for all $(\lambda, x) \in \mathbb{R}_{+} \times \mathbb{R}^{d}$ that

$$
\begin{aligned}
w(\lambda, x) & \leq \sup _{\tau \in \mathcal{T}} E\left[V\left(E\left[H_{\tau}\right], x\right)-\lambda H_{\tau}\right] \\
& \leq \sup _{T \geq 0}(V(T, x)-\lambda T)=-V^{*}(\lambda, x) .
\end{aligned}
$$

This completes the proof of the proposition.

For every $\lambda \in \mathbb{R}_{+}$the function $\mathbb{R}^{d} \ni x \mapsto w(\lambda, x) \in \mathbb{R} \cup\{+\infty\}$ is the value function of a stopping problem. One way to tackle this problem is the classical PDE approach. The associated dynamic programming equation takes for every $\lambda \in \mathbb{R}_{+}$the variational form (cf. eg. [9, Chapter IV Section 8])

$$
\min [-\mathcal{L} \tilde{w}(\lambda, x)+\lambda h(x), \tilde{w}(\lambda, x)-f(x)]=0 \quad \text { for all } x \in \mathbb{R}^{d} .
$$

Note, however, that in general the solution of this equation is not unique. This can be seen as follows. Suppose that $\left(X_{t}^{x}\right)_{t \geq 0}$ is a Brownian motion starting in $x \in \mathbb{R}^{d}$ and let $h(x)=1$ for all $x \in \mathbb{R}^{d}$. Furthermore, assume that $f: \mathbb{R}^{d} \rightarrow \mathbb{R}$ is bounded from above by some constant $c \in \mathbb{R}$. Then the functions $\tilde{w}^{r}: \mathbb{R}_{+} \times \mathbb{R}^{d} \rightarrow \mathbb{R}, \tilde{w}^{r}(\lambda, x)=\frac{\lambda}{d}\|x\|^{2}+r, r \geq c$ all satisfy Equation (6.2). Observe that if $V$ satisfies the duality relation $V=V^{* *}$, then the initial condition $V(0, \cdot)=f$ translates to the identity $f(x)=V(0, x)=$ $\inf _{\lambda \geq 0}-V^{*}(\lambda, x)=-\sup _{\lambda \geq 0} V^{*}(\lambda, x)$ for all $x \in \mathbb{R}^{d}$. This suggests to impose the further condition $\inf _{\lambda \geq 0} \tilde{w}(\lambda, x)=f(x)$ on the solutions of the PDE (6.2). This might lead to uniqueness, but renders the computation of $\tilde{w}$ more difficult.

If it is still possible to determine $w$ and if $V=V^{* *}$, then it follows from Proposition 6.1 that one can recover $V$ from $w$ via the identity $V=(-w)^{*}$. Moreover if $\tau_{\lambda, x}$ is optimal in the stopping problem (6.1) for $(\lambda, x) \in \mathbb{R}_{+} \times \mathbb{R}^{d}$ and satisfies the constraint $E\left[H_{\tau_{\lambda, x}}\right]=T$ for some $T \geq 0$, then $\tau_{\lambda, x}$ is also optimal in the problem $V(T, x)=\sup _{\tau \in \mathcal{S}(T)} E\left[f\left(X_{\tau}^{x}\right)\right]$. Indeed, for every $\tau \in \mathcal{S}(T)$ it holds that

$$
E\left[f\left(X_{\tau_{\lambda, x}}^{x}\right)\right]=w(\lambda, x)+\lambda T \geq E\left[f\left(X_{\tau}^{x}\right)-\lambda H_{\tau}\right]+\lambda T \geq E\left[f\left(X_{\tau}^{x}\right)\right] .
$$

In general, for a fixed $(\lambda, x) \in \mathbb{R}_{+} \times \mathbb{R}^{d}$ an optimal stopping time in (6.1) is the first hitting time of the stopping region, i.e. $\tau_{\lambda, x}=\inf \left\{s \geq 0 \mid \tilde{w}\left(\lambda, X_{s}^{x}\right) \leq\right.$ $\left.f\left(X_{s}^{x}\right)\right\}$. However, it may happen that there exist multiple optimal stopping times for the dual problem (6.1). In this case one has to identify the one 
matching the expectation constraint $E\left[H_{\tau_{\lambda, x}}\right]=T$ among all these optimal stopping times. In particular, it is not sufficient to determine only $\tau_{\lambda, x}$. To illustrate this fact we revisit Example 5.6, where optimal stopping times for (6.1) also include stopping times that are not first hitting times of two points.

Example 6.2 (cf. Example 5.6). Let $d=1, f: \mathbb{R} \rightarrow \mathbb{R}, f(y)=y^{2} \mathbb{1}_{\{|y| \geq 1\}}$, $h: \mathbb{R} \rightarrow \mathbb{R}, h(y)=1$ and let $X$ be a one-dimensional Brownian motion which starts in 0 . Then for the dual problem it is straightforward to show that

$$
w(\lambda, 0)=\sup _{E[\tau]<\infty} E\left[\left(\mathbb{1}_{\left\{\left|W_{\tau}\right| \geq 1\right\}}-\lambda\right) W_{\tau}^{2}\right]= \begin{cases}\infty, & \text { if } \lambda<1, \\ 0, & \text { if } \lambda \geq 1 .\end{cases}
$$

This implies that $(-w)^{*}(T, 0)=T$ for all $T \geq 0$. Therefore it follows from Section 5.6 that $V(T, 0)=(-w)^{*}(T, 0)$ for all $T \geq 0$. For $\lambda<1$ there exist no optimal stopping times and for $\lambda \geq 1$ stopping immediately $\tau=0$ is optimal. But for $\lambda=1$ all stopping times $\tau_{a}$ that embed the distribution $\frac{a}{2} \delta_{-1}+(1-a) \delta_{0}+\frac{a}{2} \delta_{1}, a \in[0,1]$ into $W$ are also optimal. It holds that $E\left[\tau_{a}\right]=a$. It follows that $\tau_{T}$ is optimal in the primal problem $V(T, 0)=$ $\sup _{\tau \in \mathcal{S}(T)} E\left[f\left(W_{\tau}\right)\right]$ for all $T \geq 0$.

\section{Examples with no optimal stopping time}

\subsection{An optimal stopping time does not exist for $U(T, 0)$}

We now present an example where no optimal stopping time exists for $U(T, 0)$, but for $U(T, x)$ if $x \neq 0$. Moreover, the value function does not solve the PDE (4.1) in $(T, 0)$. In addition, $V$ coincides with $U$ and it is a classical solution to (5.2).

Let $f(y)=e^{-|y|}, h(y)=1, y \in \mathbb{R}$, and let $X_{t}^{x}=x+W_{t}, t \geq 0$, be a Brownian motion starting in $x \in \mathbb{R}$. Then,

$$
U(T, x)= \begin{cases}1, & \text { if } x=0, \\ \frac{1}{T+x^{2}}\left(T+x^{2} e^{-|x|-\frac{T}{|x|}}\right), & \text { if } x \neq 0,\end{cases}
$$

$U \in \mathcal{C}^{2}((0, \infty) \times \mathbb{R})$, but there exists no optimal stopping time for $U(T, 0)$, $T>0$. In addition, $U$ is a solution to (4.1) on $(0, \infty) \times \mathbb{R} \backslash\{0\}$ but only a strict supersolution on $(0, \infty) \times\{0\}$ :

$$
h(x) U_{T}(T, x)-\frac{1}{2} U_{x x}(T, x)+\frac{1}{2} \frac{U_{T x}^{2}(T, x)}{U_{T T}(T, x)}= \begin{cases}0, & \text { if } x \neq 0, \\ \frac{1}{T}, & \text { if } x=0 .\end{cases}
$$

We first show that $U(T, 0)=1$ and that there does not exist an optimal stopping time for $U(T, 0)$. To see this, let $T>0$ and notice that the first 
exit time $\sigma(T, \varepsilon)$ of $(-\varepsilon, T / \varepsilon), \varepsilon>0$, has expectation $T$. Thus,

$$
U(T, 0) \geq \sup _{\varepsilon>0} E\left[f\left(X_{\sigma(T, \varepsilon)}^{0}\right)\right]=\sup _{\varepsilon>0}\left\{\frac{T e^{-\varepsilon}}{T+\varepsilon^{2}}+\frac{\varepsilon e^{-\frac{T}{\varepsilon}}}{T+\varepsilon^{2}}\right\}=1 .
$$

Since $f$ is bounded above by 1 , we conclude that $U(T, 0)=1$. Now assume that there exists an optimal stopping time $\tau^{*}$ for $U(T, 0)$ with $T>0$, i.e. $E\left[f\left(X_{\tau^{*}}^{0}\right)\right]=1$ and $E\left[\tau^{*}\right]=T$. This implies $X_{\tau^{*}}^{0}=0$ a.s., but this is not possible if $\tau^{*}$ is integrable with $E\left[\tau^{*}\right]>0$. Hence, an optimal stopping time for $U(T, 0), T>0$, does not exist.

Now let $x \neq 0$. For $T>0$ the reward of the first exit time $\sigma_{x}(0, x+T / x)$ of $(0, x+T / x), x>0$, resp. $(x+T / x, 0), x<0$, is given by

$$
E\left[f\left(X_{\sigma_{x}(0, x+T / x)}^{x}\right)\right]=\frac{1}{T+x^{2}}\left(T+x^{2} e^{-|x|-\frac{T}{|x|}}\right)=: u(T, x) .
$$

It holds true that $u \in \mathcal{C}^{2}((0, \infty) \times \mathbb{R})$ and $u$ is a solution to $(4.1)$ on $(0, \infty) \times$ $\mathbb{R} \backslash\{0\}$. But for $T>0$ we have

$$
h(x) u_{T}(T, 0)-\frac{1}{2} u_{x x}(T, 0)+\frac{1}{2} \frac{u_{T x}^{2}(T, 0)}{u_{T T}(T, 0)}=0-\frac{1}{2}\left(-\frac{2}{T}\right)+\frac{1}{2} 0=\frac{1}{T} .
$$

Hence, $u$ is a supersolution to (4.1). Moreover, it is concave with $u_{T T}(T, x)<$ 0 for $x \neq 0$ and $u_{T x}(T, x)=0$ on $\left\{(T, x) \in(0, \infty) \times \mathbb{R} \mid u_{T T}(T, x)=0\right\}=$ $(0, \infty) \times\{0\}$. Since $|u|$ is bounded above $1, u$ satisfies the growth condition (5.1) with $p=1$. Theorem 5.1 implies that $u$ dominates the value function and, therefore, $U=u$.

Remember that the quotient $U_{T x}^{2} / U_{T T}$ is set to 0 if the nominator and the denominator equal 0 . Here we have

$$
\frac{U_{T x}^{2}(T, x)}{U_{T T}(T, x)} \underset{x \rightarrow 0}{\longrightarrow}-\frac{2}{T} \neq 0 .
$$

Hence, $(T, x) \mapsto U_{T x}^{2}(T, x) / U_{T T}(T, x)$ is not continuous on $(0, \infty) \times\{0\}$ and, therefore, $U$ cannot be a solution to (4.1) by Remark 4.2(b).

It is worth mentioning that $\tau^{*}=0$ is an optimal stopping time for $V(T, 0)$. Moreover, $V(T, x)=U(T, x)$, because $U_{T}(T, x)>0$ for $x \neq 0$. In particular, $V$ is a solution to $(5.2)$ on the whole domain $(0, \infty) \times \mathbb{R}$.

\subsection{Maximizing a concave payoff function}

We now that show if $X$ is a martingale and $f$ a concave payoff function, then the value function $U$ is only a strict supersolution to (4.1). Moreover, $U$ is independent of $T$ and coincides with $f$.

Let $n=d=1, h(y)=1, y \in \mathbb{R}, \mu=0$ and $\sigma(x) \geq \delta>0$. Then, for all $x \in \mathbb{R}$ the process $\left(X_{t}^{x}\right)$ is a martingale with $\left\langle X^{x}, X^{x}\right\rangle_{\infty}=\infty$, 
a.s. For every concave and continuous $f$ with subquadratic growth, i.e. $\lim _{y \rightarrow \pm \infty}|f(y)| / y^{2}=0$, the value functions $U$ and $V$ are given by

$$
U(T, x)=V(T, x)=f(x)
$$

for all $(T, x) \in[0, \infty) \times \mathbb{R}$. On the one hand the function $f(x)$ dominates $U(T, x)$ and $V(T, x)$ by Jensen's inequality. To prove the reverse inequality, we define for every $(T, x) \in(0, \infty) \times \mathbb{R}$ and $\varepsilon>0$ the stopping times

$$
\sigma_{x}(\varepsilon, T)=\inf \left\{t \geq 0 \mid X_{t}^{x} \notin\left(x-\varepsilon, x+\frac{T}{\varepsilon}\right)\right\} .
$$

Notice that $E\left(\sigma_{x}(\varepsilon, T)\right)=T$, and hence we have

$$
\begin{aligned}
U(T, x) & \geq \sup _{\varepsilon>0} E\left[f\left(X_{\sigma_{x}(\varepsilon, T)}^{x}\right)\right] \\
& =\sup _{\varepsilon>0}\left\{f\left(x+\frac{T}{\varepsilon}\right) \frac{\varepsilon^{2}}{T+\varepsilon^{2}}+f(x-\varepsilon) \frac{T}{T+\varepsilon^{2}}\right\} \\
& \geq \lim _{\varepsilon \rightarrow 0}\left\{f\left(x+\frac{T}{\varepsilon}\right) \frac{\varepsilon^{2}}{T+\varepsilon^{2}}+f(x-\varepsilon) \frac{T}{T+\varepsilon^{2}}\right\} \\
& =f(x) .
\end{aligned}
$$

If, in addition, $f$ is twice continuously differentiable and there exists $y \in \mathbb{R}$ with $f^{\prime \prime}(y)<0$, then $U$ is not a solution to (4.1) on $(0, \infty) \times\left\{y \in \mathbb{R} \mid f^{\prime \prime}(y)<\right.$ $0\}$. Notice, however, $V$ is a solution to $(5.2)$ on $(0, \infty) \times \mathbb{R}^{n}$.

\subsection{The value function is linear in $T$}

In the previous two examples the value function $U$ does not solve the PDE (4.1), but $V$ is a solution to (5.2). We now present an example where both $U$ and $V$ do not solve the corresponding dynamic programming equation.

Let $f(y)=y^{2}-\sqrt{1+y^{2}}, h(y)=1, y \in \mathbb{R}$, and let $X_{t}^{x}, t \geq 0$, be a onedimensional Brownian motion with $X_{0}^{x}=x \in \mathbb{R}$. For every stopping time $\tau \in \mathcal{S}(T), T>0$, we have

$E\left[f\left(X_{\tau}^{x}\right)\right]=x^{2}+E[\tau]+E\left[-\sqrt{1+\left(X_{\tau}^{x}\right)^{2}}\right] \leq x^{2}+T-\sqrt{1+x^{2}}=T+f(x)$

by Jensen's inequality. Hence, $U(T, x) \leq V(T, x) \leq T+f(x)$.

As in Example 7.2 we define for every $(T, x) \in(0, \infty) \times \mathbb{R}$ and $\varepsilon>0$ the stopping times

$$
\sigma_{x}(\varepsilon, T)=\inf \left\{t \geq 0 \mid X_{t}^{x} \notin\left(x-\varepsilon, x+\frac{T}{\varepsilon}\right)\right\} .
$$


Then it follows that

$$
\begin{aligned}
& U(T, x) \geq \sup _{\varepsilon>0} E\left[f\left(X_{\sigma_{x}(\varepsilon, T)}^{x}\right)\right] \\
& =\sup _{\varepsilon>0}\left\{\left(\left(x+\frac{T}{\varepsilon}\right)^{2}-\sqrt{1+\left(x+\frac{T}{\varepsilon}\right)^{2}}\right) \frac{\varepsilon^{2}}{T+\varepsilon^{2}}+\left((x-\varepsilon)^{2}-\sqrt{1+(x-\varepsilon)^{2}}\right) \frac{T}{T+\varepsilon^{2}}\right\} \\
& =T+f(x) .
\end{aligned}
$$

Therefore, $V(T, x)=U(T, x)=T+f(x)$.

$U$ is not a solution to (4.1), because

$$
h(x) U_{T}(T, x)-\mathcal{L} U(T, x)+\frac{U_{T x}^{2}(T, x)}{2 U_{T T}(T, x)}=\frac{1}{2\left(1+|x|^{2}\right)^{3 / 2}}>0 .
$$

Moreover, $V$ does not solve the PDE (5.2). Indeed,

$\min \left\{V_{T}(T, x), h(x) V_{T}(T, x)-\mathcal{L} V(T, x)+\frac{V_{T x}^{2}(T, x)}{2 V_{T T}(T, x)}\right\}=\min \left\{1, \frac{1}{2\left(1+|x|^{2}\right)^{3 / 2}}\right\}>0$.

\section{References}

[1] O. Bokanowski, A. Picarelli, and H. Zidani. State-constrained stochastic optimal control problems via reachability approach.

[2] B. Bouchard, R. Elie, and N. Touzi. Stochastic target problems with controlled loss. SIAM J. Control Optim., 48(5):3123-3150, 2009/10.

[3] B. Bouchard and N. Touzi. Weak dynamic programming principle for viscosity solutions. SIAM J. Control and Optimization, 49(3):948-962, 2011.

[4] R. Durrett. Probability: theory and examples. Cambridge Series in Statistical and Probabilistic Mathematics. Cambridge University Press, Cambridge, fourth edition, 2010.

[5] M. Horiguchi. Markov decision processes with a stopping time constraint. Math. Methods Oper. Res., 53(2):279-295, 2001.

[6] I. Karatzas and S. E. Shreve. Brownian motion and stochastic calculus, volume 113 of Graduate Texts in Mathematics. Springer-Verlag, New York, second edition, 1991.

[7] D. P. Kennedy. On a constrained optimal stopping problem. J. Appl. Probab., 19(3):631-641, 1982.

[8] J. Palczewski and E. Stettner. Infinite horizon stopping problems with (nearly) total reward criteria. Stochastic Process. Appl., 124(12):38873920, 2014.

[9] G. Peskir and A. Shiryaev. Optimal stopping and free-boundary problems. Springer, 2006. 